\title{
Angiokine Wisp-1 is increased in myocardial infarction and regulates cardiac endothelial signaling
}

\author{
Lillianne H. Wright, ${ }^{1}$ Daniel J. Herr, ${ }^{1}$ Symone S. Brown, ${ }^{2}$ Harinath Kasiganesan, ${ }^{1}$ \\ and Donald R. Menick ${ }^{1,3}$ \\ 'Division of Cardiology, and ${ }^{2}$ College of Graduate Studies, Summer Undergraduate Research Program, Medical University of \\ South Carolina, Charleston, South Carolina, USA. ${ }^{3}$ Ralph H. Johnson VA Medical Center, Charleston, South Carolina, USA.
}

\begin{abstract}
Myocardial infarctions (MIs) cause the loss of myocytes due to lack of sufficient oxygenation and latent revascularization. Although the administration of histone deacetylase (HDAC) inhibitors reduces the size of infarctions and improves cardiac physiology in small-animal models of MI injury, the cellular targets of the HDACs, which the drugs inhibit, are largely unspecified. Here, we show that WNT-inducible secreted protein-1 (Wisp-1), a matricellular protein that promotes angiogenesis in cancers as well as cell survival in isolated cardiac myocytes and neurons, is a target of HDACs. Further, Wisp- 1 transcription is regulated by HDACs and can be modified by the HDAC inhibitor, suberanilohydroxamic acid (SAHA/vorinostat), after MI injury. We observe that, at 7 days after MI, Wisp-1 is elevated 3 -fold greater in the border zone of infarction in mice that experience an MI injury and are injected daily with SAHA, relative to MI alone. Additionally, human coronary artery endothelial cells (HCAECs) produce WISP-1 and are responsive to autocrine WISP-1-mediated signaling, which functionally promotes their proangiogenic behavior. Altering endogenous expression of WISP-1 in HCAECs directly impacts their network density in vitro. Therapeutic interventions after a heart attack define the extent of infarct injury, cell survival, and overall prognosis. Our studies shown here identify a potentially novel cardiac angiokine, Wisp-1, that may contribute to beneficial post-MI treatment modalities.
\end{abstract}

Conflict of interest: The authors have declared that no conflict of interest exists.

Submitted: June 22, 2017

Accepted: January 10, 2018

Published: February 22, 2018

Reference information:

JCI Insight. 2018;3(4):e95824. https:// doi.org/10.1172/jici.insight.95824.

\section{Introduction}

Over 700,000 people in the US each year will experience a myocardial infarction (MI) (1). MIs occur when a major coronary artery becomes blocked. This blockage results in an acute ischemic injury that leads to localized myocyte cell death due to the lack of sufficient oxygenation (2-4). Adverse tissue remodeling after an infarction due to necrosis, inflammation, and excessive fibrosis can eventually lead to impaired cardiac physiology and heart failure (4-6). Several groups have demonstrated the benefits of reestablishing and or sustaining vasculature peripherally to infarcted myocardium, which results in attenuated myocyte cell death $(2,7,8)$ in animal models. Despite these promising data, dynamic changes in post-MI myocardium over time have challenged traction in establishing beneficial angiogenesis throughout infarct healing.

The extent of infarct expansion after an acute MI injury is greatly impacted by secreted proteins in the microenvironment, especially in the border-zone tissue surrounding the injury. Secreted proteins regulate a variety of cellular processes after infarction, including collagen deposition, cell survival, and chemokine-mediated recruitment of cells to the injury (5,9-11). One family of secreted matricelluar proteins, collectively termed CCNs (cysteine rich angiogenic inducer-61, connective TGF, nephroblastoma overexpressed, WNT inducible secreted protein-1,2 and 3 [Wisp-1, -2, and-3]), have been shown to greatly impact pathological and nonpathological processes by directly mediating tissue microenvironments.

In the context of cardiovascular biology, Colston et al. observed that Wisp-1, is upregulated in response to an MI in animal models (12). Additionally, they demonstrated that recombinant Wisp-1 (rec.Wisp-1) protein treatment of isolated myocytes and fibroblasts promotes cell survival, in response to cytokine stimulation, and collagen secretion of fibroblasts (12). However, their studies did not explore 
End Diastolic Volume at 7 Days Post-MI
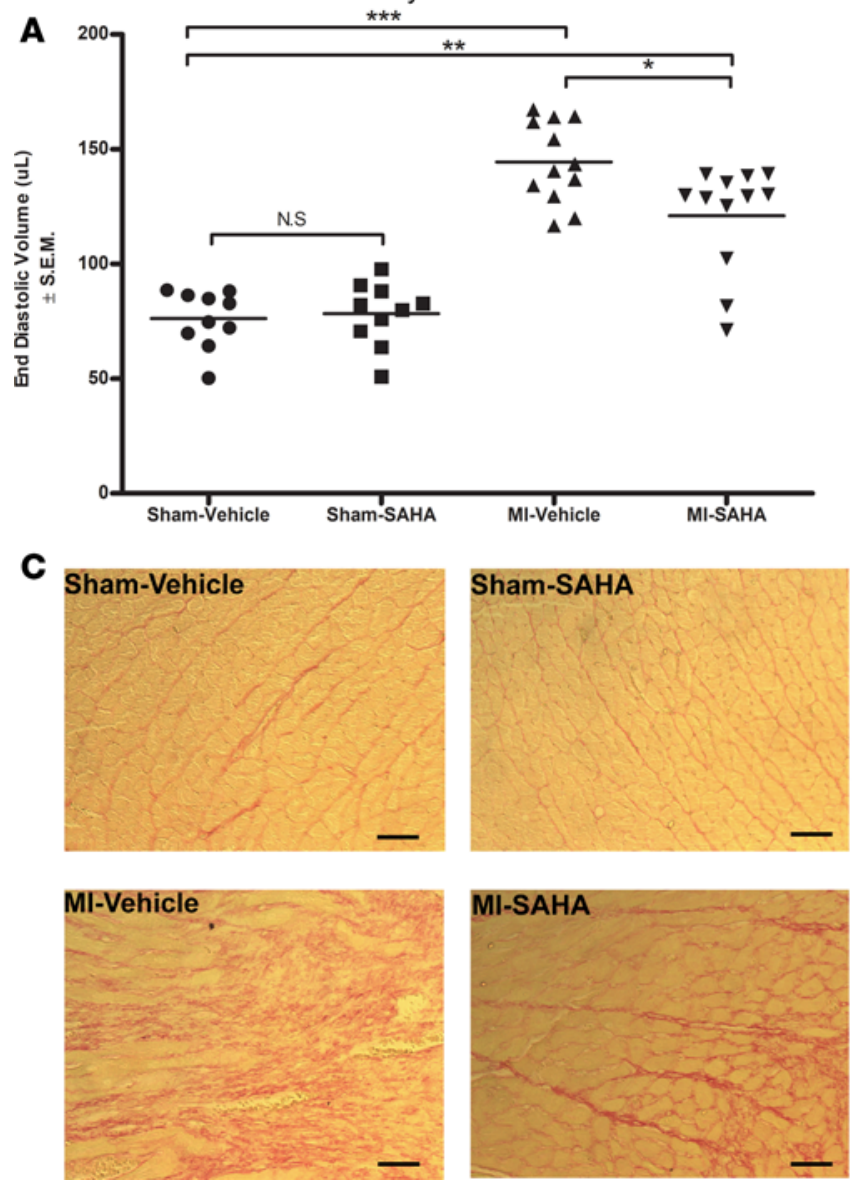

Ejection Fraction at 7 Days Post-MI

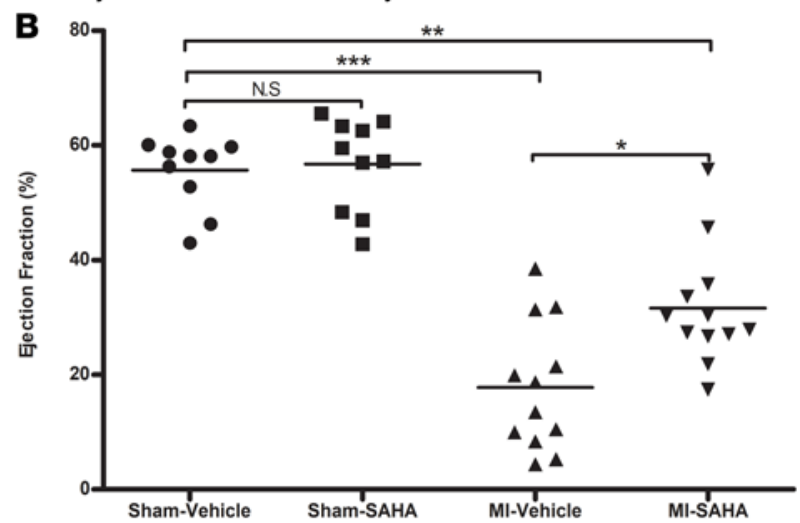

Collagen Density in LV at 7 Days Post-MI

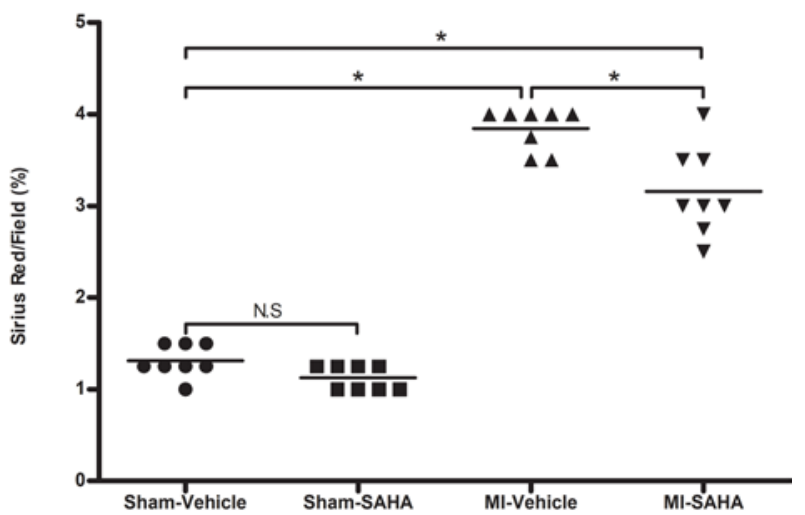

Figure 1. Inhibition of HDACs improves cardiac physiology at 7 days after MI. Ten- to 12-week-old male CD1 mice were subjected to either sham (control) or ligation of the coronary artery surgery and received daily i.p. injections of DMSO (vehicle-control) or the HDAC inhibitor SAHA (25 mg/kg) for 7 days after MI. (A) End diastolic volume and (B) ejection fraction. (C) Sirius red, a marker of collagen. Magnification, 20x; scale bars: $10 \mu \mathrm{m}$. A and B are from 10 mice/ sham and 12 mice/Ml group and are representative of 2 independent studies. $\mathbf{C}$ is from 8 mice per group and is representative of 2 experiments. Results depicted as mean $\pm \mathrm{SEM},{ }^{*} P \leq 0.05,{ }^{* *} P \leq 0.01,{ }^{* * *} P \leq 0.001$. $P$ values obtained by 1 -way ANOVA with Tukey's post test.

what role Wisp-1 might be playing in post-MI myocardium in vivo. Given the fact that Wisp-1 promotes angiogenesis in colon, breast, and oral squamous cancers (13-15) and cell survival in normoxic and ischemic neurological pathologies $(16,17)$, we wanted to explore whether changes in the expression of Wisp-1 might be playing an important role in postinfarction myocardium.

Mechanistically, a group of enzymes called histone deacetylases (HDACs) play integral roles in the regulation of gene expression, protein localization, and protein stability in cardiovascular diseases through deacetylation of histone and nonhistone proteins (18-20). Specifically, HDACs remove acetyl groups from lysine residues (21), which is why nomenclature is shifting from HDAC to the more inclusive/comprehensive role that these enzymes play as KDACs. There are 18 known HDACs, which are subdivided into 4 classes (I, II, III, and IV) by structural homology, enzymatic activity, and subcellular localization (21-23). We and others have observed that small molecule HDAC inhibitors that target class I HDACs, which include HDACs 1-3, attenuate cell death, reduce myocardial areas of infarction, and promote improved cardiovascular function in small-animal models (24-27). We know very little about which targets of HDACs are directly contributing to this improvement.

Wisp-1 is potently upregulated by transcriptional coactivators $\beta$-catenin and $\mathrm{T}$ cell factor/lymphoid enhancer factor (TCF/LEF) (28). $\beta$-Catenin can also be acetylated and, once acetylated, has a higher affinity for both chromatin and TCF/LEF-activating complexes (29). Therefore, we hypothesize that Wisp-1/ Wisp-1 is increased in response to HDAC inhibition after MI and contributes to beneficial cardiac-specific angiogenesis. Our data elucidates a potentially novel target of HDACs, Wisp-1, whose expression and 
A sham-Vehicle
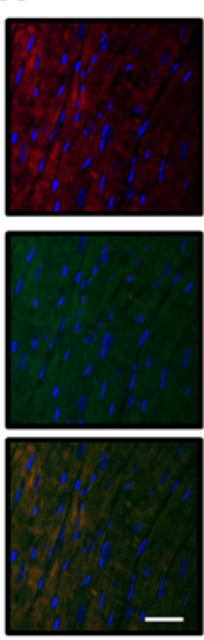

Top Panel : $\alpha$-SARCIDAP
Sham-SAHA
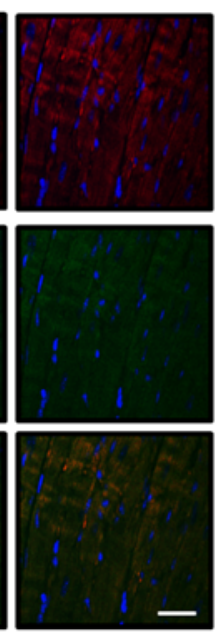
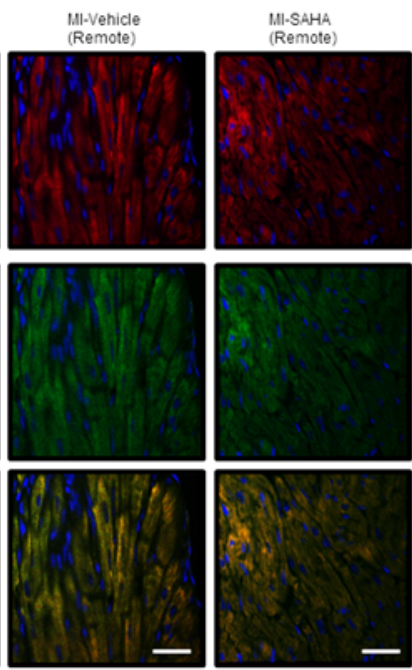

Middle Pan

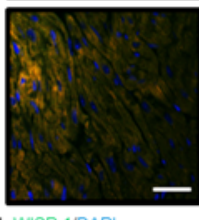

WISP-1/DAP

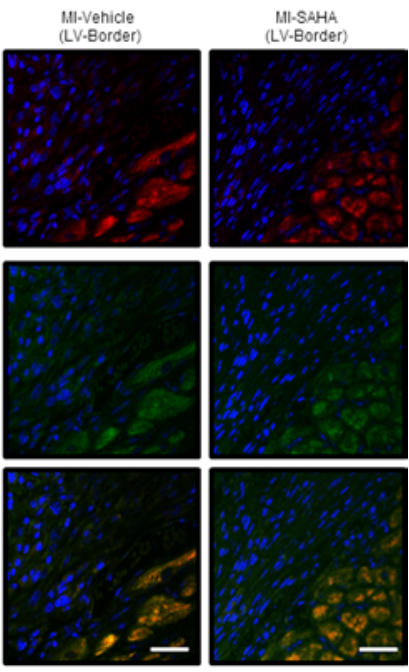

Bottom Panel:a-SARCNISP-1/DAPI

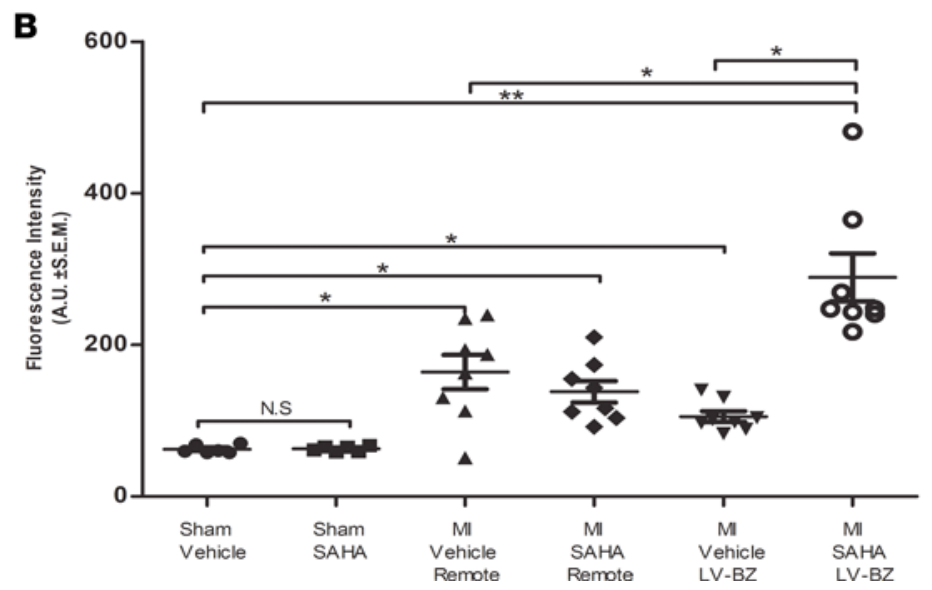

Figure 2. Wisp-1 is upregulated in response to MI injury and HDAC inhibition at the border zone of infarction 7 days after MI. Ten- to 12 -week-old male CD1 mice were subjected to either sham (control) or ligation of the coronary artery surgery and received daily i.p. injections of DMSO (vehicle-control) or the HDAC inhibitor SAHA (25 mg/kg) for 7 days after MI. (A) Immunofluorescent image of post-MI myocardium. Wisp-1 and the skeletal muscle marker, $\alpha$-sarcomeric (red/TRITC), using immunostaining. Magnification, 20x; scale bars: $100 \mu \mathrm{m}$. (B) Relative fluorescence intensity (green/FITC) per field was determined using Image J (Fiji) and normalized to nuclei (DAPI/blue) and was quantified by AUs. A and B are from 10 mice/sham and 12 mice/MI group and are representative of 2 independent studies. Results depicted as mean $\pm S E M,{ }^{*} P \leq 0.05$, ${ }^{* *} P \leq 0.01$. $P$ values obtained by 1-way ANOVA with Tukey's post test.

abundance are mediated through suberanilohydroxamic acid (SAHA) in post-MI myocardium. Our data also elucidate that WISP-1 directly regulates human cardiac-specific endothelial cells and is therefore a potential pharmacological target for revascularization after an acute ischemic cardiac injury.

\section{Results}

$H D A C$ inhibition enhances the expression and abundance of Wisp-1 in Left ventricle (LV) border regions at 7 days after MI. To determine whether HDAC inhibition impacts the expression and or abundance of Wisp-1 after MI, we ligated the left anterior descending (LAD) coronary artery of 10- to 12-week-old male mice to induce an acute MI injury. Twelve hours after surgery, mice were injected (i.p.) daily with either vehicle (DMSO) or an HDAC inhibitor, SAHA ( $5 \mathrm{mg} / \mathrm{kg}$ ), for 7 days; treatment and dosage used was based upon previous investigations (30-33). We also performed sham operations and subsequent daily vehicle or SAHA i.p. injections in a separate cohort of mice to serve as procedural controls. On the seventh day after MI, we assessed cardiac physiology just before euthanasia and then harvested cardiac tissue to assess fibrosis and Wisp-1/Wisp-1.

Consistent with previous studies $(20,32)$, HDAC inhibition improved cardiac physiology by reducing end diastolic volume and increasing ejection fraction (Figure 1, A and B) relative to MI-vehicle. Collagen deposition was reduced by approximately $12 \%$ in post-MI mice that were treated with SAHA, relative to 

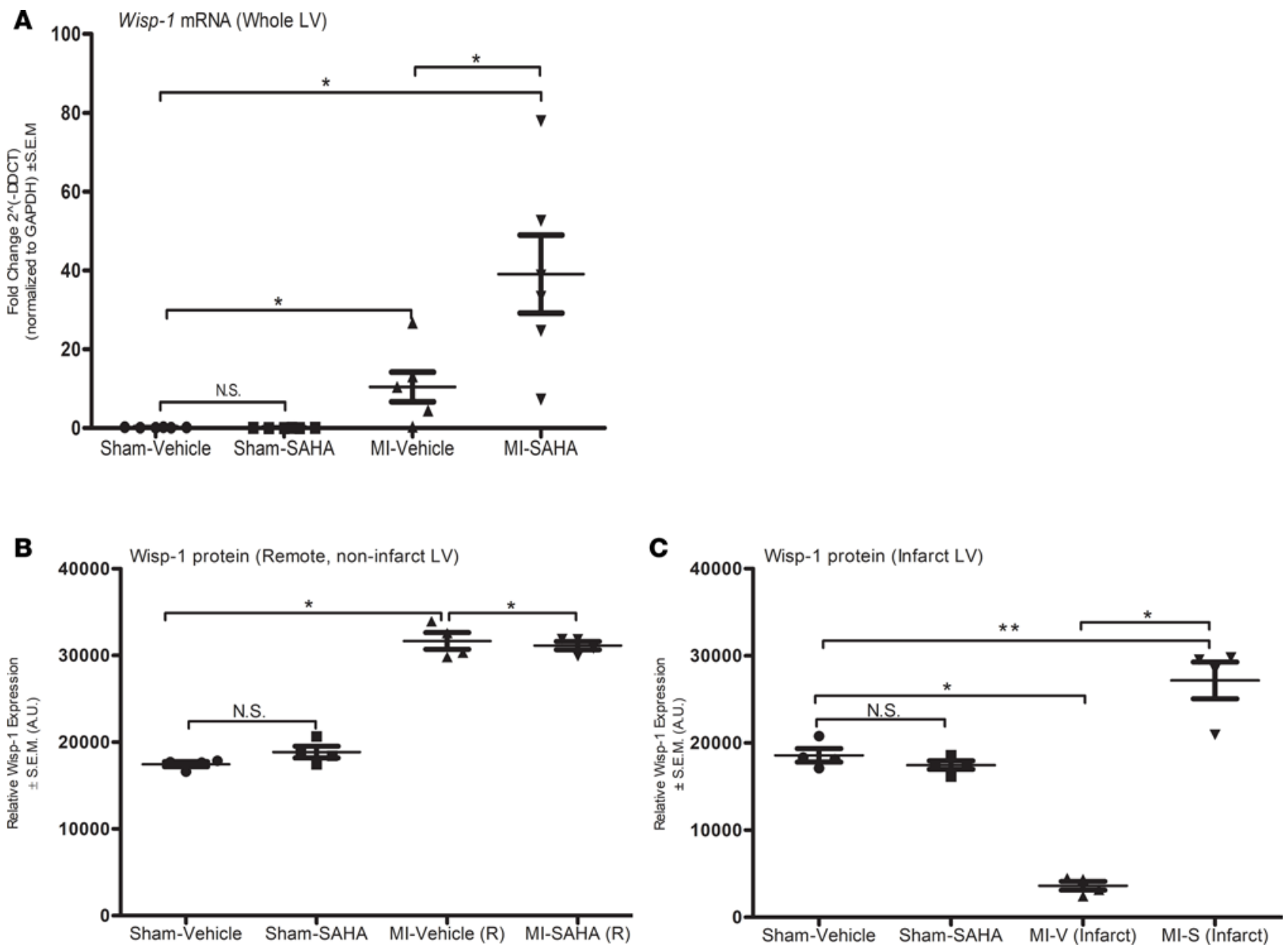

Figure 3. Wisp-1 mRNA and Wisp-1 protein expression are increased in response to MI injury and HDAC inhibition within the LV 7 days after MI. (A) qPCR of Wisp-1 expression from LV at 7 days after MI. Data represents the $2^{-\Delta \Delta c t}$ and are normalized to Gapdh CT values. Western blot analysis of Wisp-1 protein in (B) remote noninfarct LV and (C) infarcted LV of post-MI myocardium or sham control tissue. Data are normalized to Gapdh. A is from 6 mice per group. qPCR was performed in triplicated and repeated 3 times. $\mathbf{B}$ and $\mathbf{C}$ are from 4 mice per group and representative of 3 independent studies. Results depicted as mean $\pm \mathrm{SEM}$. ${ }^{*} P \leq 0.05,{ }^{*} P \leq 0.01$. $P$ values obtained by 1 -way ANOVA with Tukey's post test.

MI alone at 7 days after MI (Figure 1C). Semiquantitative analyses of microscopy images (Figure 2A) determined that MI injury alone significantly induced Wisp-1 abundance approximately 2 - to 2.5 -fold in remote, noninfarcted ventricle of both MI-vehicle- and MI-SAHA-treated mice relative to sham (Figure 2B). In the LV border zones of infarction (LV-border), we observed a 2-fold increase of Wisp-1 in MI-vehicle, similar to that of remote tissue. However, MI-SAHA-treated mice demonstrated a 5-fold increase of detectable Wisp-1 relative to sham controls (Figure 2B).

We further analyzed Wisp-1 in post-MI myocardium by quantifying Wisp-1/Wisp-1 expression within various regions of LV. Wisp-1 mRNA is significantly upregulated 15-fold in MI-vehicle (LV) and almost 45-fold in MI-SAHA (LV) relative sham (Figure 3A). In parallel, we also assessed whole-LV tissue homogenates to assess tubulin acetylation to validate the efficacy of HDAC inhibition/SAHA (Supplemental Figure 1A; supplemental material available online with this article; https://doi.org/10.1172/jci. insight.95824DS1) and Wisp-1 abundance (Supplemental Figure 1B). At 7 days after MI, data demonstrated that, as with its mRNA, MI injury alone was sufficient to increase Wisp-1 protein in both MI-vehicleand MI-SAHA-treated cohorts. However, Wisp-1 protein was slightly increased in whole-LV lysates from MI-SAHA mice relative to MI-vehicle ( $n=4$ /group, $P \leq 0.05$ ). In order to further assess Wisp- 1 abundance in more specific regions of post-MI ventricle, we harvested tissue from infarcted LV and remote noninfarct LV tissue. Remote tissue, as with whole-LV MI homogenates, showed that Wisp-1 was significantly increased in both MI-vehicle- and MI-SAHA-treated cohorts, relative to sham (Figure 3B). However, 

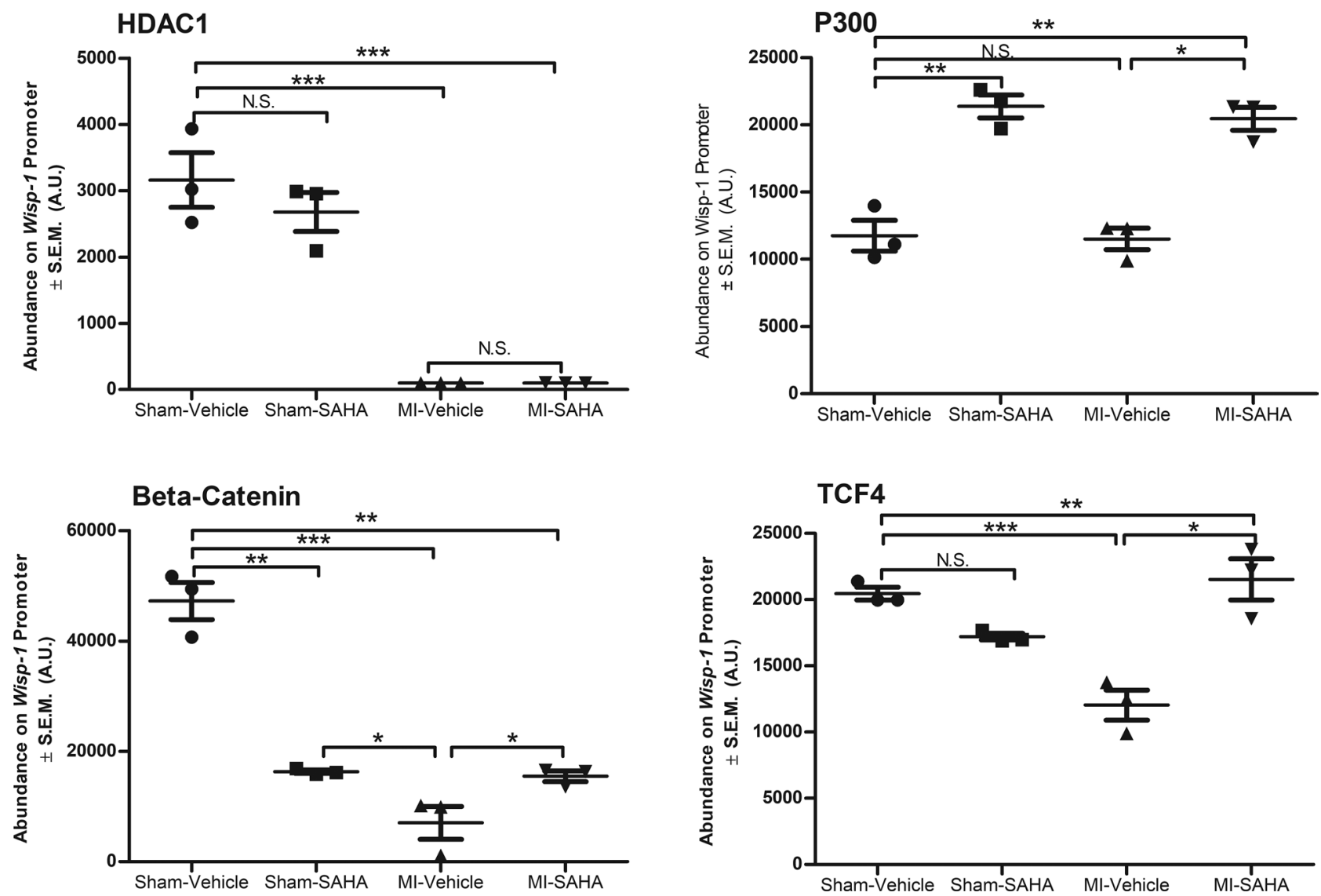

Figure 4. Inhibition of HDACs enhances recruitment and retention of Wisp-1 transcriptional regulators. Ten- to 12-week-old male CD1 mice were subjected to either sham (control) or ligation of the coronary artery surgery and received daily i.p. injections of DMSO (vehicle-control) or the HDAC inhibitor SAHA $(25 \mathrm{mg} / \mathrm{kg}$ ) for 7 days after MI. ChIP analyses of left ventricle homogenates harvested from sham and MI experimental groups for HDAC1, P300, $\beta$-catenin, and TCF4 on the proximal promoter of Wisp-1. Data are from 4 mice per group, and experiments were repeated 3 times. Results depicted as mean \pm SEM, ${ }^{*} P \leq 0.05,{ }^{* *} P \leq 0.01,{ }^{* *} P \leq 0.001$. $P$ values obtained by 1-way ANOVA with Tukey's post test.

Wisp-1 was down almost 5-fold in MI-vehicle infarcted tissue, while MI-SAHA-treated mice showed an abundance of Wisp-1 that significantly exceeds sham levels (Figure 3C).

We next used ChIP to determine whether HDAC inhibition impacts the recruitment and or retention of transcriptional regulatory elements within the proximal promoter $(-175$ through +175 relative to transcriptional start site) of Wisp-1 in whole-LV tissue at 7 days after MI. We chose antibodies directed against class I HDACs, since previous studies demonstrate they possess the greatest deacetylase activity $(19,34,35)$ and SAHA pharmacologically inhibits them. We also assessed the impact of HDAC inhibition on the histone acetyltransferase (HAT), P300, since it is an endogenous antagonist of HDACs through its enzymatic addition of acetyl groups on lysine residues (reviewed in ref. 36). Lastly, we assessed the impact of HDAC inhibition on the recruitment of transcription factors $\beta$-catenin and TCF, since they form a regulatory complex that upregulates Wisp-1; further, their interactions are sustained longer by acetylation via P300 (28, 37, 38). Importantly, the region of the Wisp-1 promoter that we identified had a TCF/LEF consensus binding site $\left(5^{\prime}\right.$ - CTTTGACAA - 3') at -137 bp upstream of the transcriptional start site.

HDAC 1 abundance was significantly reduced in the proximal promoter of Wisp-1 upon MI injury, while SAHA treatment alone promoted recruitment of HDAC2 and P300 to the promoter (Figure 4). HDAC 3 was only detected on the Wisp-1 promoter in sham-SAHA and MI-vehicle LV (Supplemental Figure 2, A and B). However, in the background of both MI injury and SAHA treatment, we observed that P300 was significantly detected, as well as $\beta$-catenin and TCF relative to MI-vehicle-treated mice (Figure 4). We also assessed whether nuclear localization of $\beta$-catenin is impacted by inhibition of HDACs, since others have reported that posttranslation modifications can impact its subcellular location (reviewed in ref. 39). MI alone significantly reduced the amount of detectable $\beta$-catenin in the nuclear fraction harvested from LV, but we 

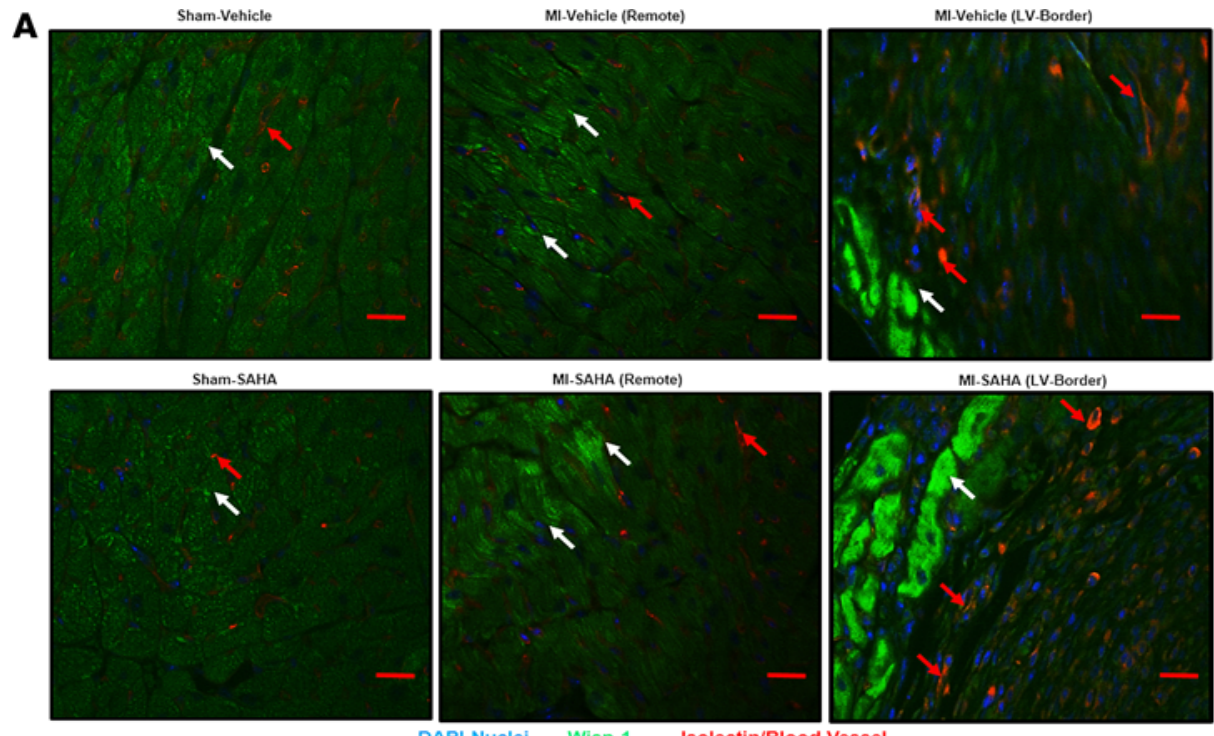

B

Isolectin Abundance 7 Days Post-MI

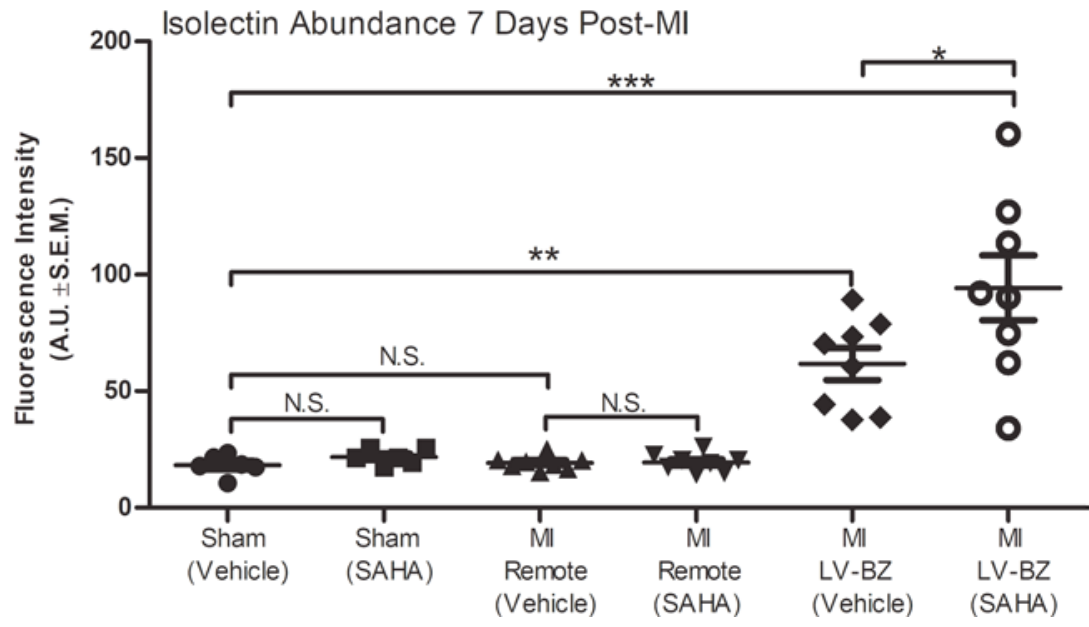

Figure 5. Wisp-1 expression is proximal to enhanced micro-vessel density at the border zone 7 days after MI. Ten- to 12-week-old male CD1 mice were subjected to either sham (control) or ligation of the coronary artery surgery and received daily i.p. injections of DMSO (vehicle-control) or HDAC inhibition, SAHA (25 mg/kg). Seven days after MI, mice were euthanized. (A) Localization and abundance of Wisp-1 (white arrows) and microvasculature (red arrows), $10 \times$ magnification, scale bars: $20 \mu \mathrm{m}$. (B) Relative fluorescence intensity (green/FITC) per field was determined using Image J (Fiji), normalized to nuclei (DAPI/blue) and quantified by AUs. A and $\mathbf{B}$ are from 6 mice/sham and 8 mice/MI group and are representative of 2 independent studies. Results depicted as mean $\pm \mathrm{SEM},{ }^{*} P \leq 0.05,{ }^{* *} P \leq 0.01,{ }^{* * *} P \leq 0.00$. $P$ values obtained by 1 -way ANOVA with Tukey's post test.

found no difference between MI-vehicle- and MI-SAHA-treated mice (Supplemental Figure 3). Together, these data suggest that Wisp-1 is a target of HDACs and can be regulated, at least transcriptionally, through inhibition HDACs within the post-MI myocardium.

Wisp-1 is proximal to increased microvasculature at the border zone at 7 days after $M I$ and promotes production of Vegf-a in cardiac cells. Wisp-1, a secreted protein, was first discovered to promote fibrovascularized regions in colon tumors and was later found to promote angiogenesis in oral cancers by upregulating Vegf-a $(14,15)$. Therefore, we asked if post-MI HDAC inhibition-mediated upregulation of Wisp-1 corresponds to increased microvasculature, and if so, where? Wisp-1 protein and blood vessel density (detected with isolectin) in both MI-vehicle and MI-SAHA were elevated at the border zones relative to sham (Figure 5A). Furthermore, semiquantitative analyses of microscopy images showed a significant elevation of blood vessel density in the MI-SAHA relative to MI-vehicle within the border zone (Figure $5 \mathrm{~B}$ ). We next assessed Vegf-a in post-MI myocardium. Vegf-a protein expression was slightly decreased in remote, noninfarcted LV due to MI injury (Figure 6A). In parallel to Wisp-1 protein expression, however, Vegf-a was significantly elevated in infarct LV of MI-SAHA-treated mice relative to MI-vehicle (Figure 6B). 

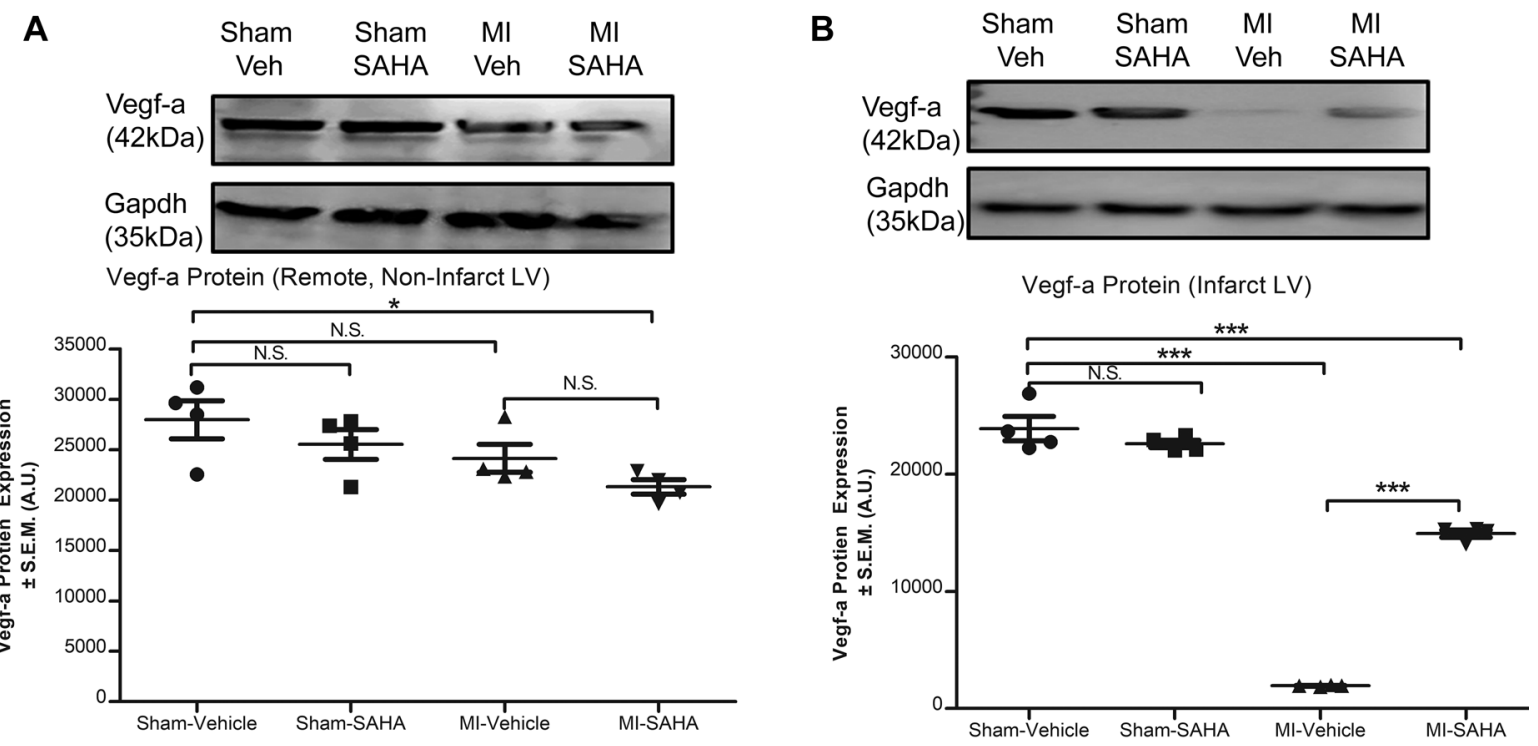

Figure 6. Vegf-a is increased in infarct LV with SAHA treatment at 7 days after MI. Ten- to 12-week-old male CD1 mice were subjected to either sham (control) or ligation of the coronary artery surgery and received daily i.p. injections of DMSO (vehicle-control) or HDAC inhibition, SAHA (25 mg/kg). Seven days after MI, mice were euthanized. Western blot analysis of Vegf-a protein from (A) remote noninfarct LV and (B) infarcted LV of post-MI myocardium or sham control tissue. Data are normalized to Gapdh. $\mathbf{A}$ and $\mathbf{B}$ are from 4 mice per group and representative of 3 independent studies. Results depicted as mean $\pm \mathrm{SEM},{ }^{*} P \leq 0.05,{ }^{* *} P \leq 0.001$. $P$ values obtained by 1-way ANOVA with Tukey's post test.

It is unclear whether the increased Vegf-a protein expression in infarcted LV was due to HDAC inhibition or due to Wisp-1 directly, as it occurs in certain cancers (14). Therefore, we isolated cardiomyocytes, cardiac fibroblasts, and endothelial cells from the LV of healthy WT mice and assessed their endogenous production of Wisp-1. Cardiac myocytes expressed the greatest relative amount of Wisp-1 protein, followed by endothelial cells, and the least was produced by fibroblasts (Figure 7A). We then assessed whether treating isolated cardiac-derived cells with rec.Wisp-1 impacts their production of Vegf-a. Treatment with rec.Wisp-1 caused a significant, dose-dependent increase of Vegf-a in myocytes (Figure 7B). In cardiac fibroblasts, we observed less sensitivity to Wisp-1, only showing an increase of Vegf-a at the $20 \mathrm{ng} / \mathrm{ml} \mathrm{con-}$ centration (Figure 7B). Interestingly, murine cardiac endothelial cells demonstrated a 2.6-fold upregulation of Vegf-a in response to rec.Wisp-1 at all concentrations (Figure 7B). Cardiac myocytes, fibroblasts, and endothelial cells endogenously expressed Wisp-1 in vitro, and treating these cells with additional Wisp-1 directly stimulated their production of Vegf-a. Each cardiac cell type that we assessed, therefore, has the potential to promote a more proangiogenic microenvironment upon MI injury.

We also assessed whether class-specific HDAC inhibition impacts Wisp-1 and Vegf-a abundance within the LV at 7 days after MI. We used the class I-specific HDAC inhibitor Entinostat (also known as MS-275; daily i.p. injection at $5 \mathrm{mg} / \mathrm{kg}$ ). MS-275 was selected since it has great specificity for inhibiting HDAC 1 , -2 , and $-3(40,41)$. Also, our ChIP data demonstrate that HDACs 1 and -2 were differentially recruited to the Wisp-1 promoter upon infarction or HDAC inhibition with SAHA (Figure 4 and Supplemental Figure 2B). Lastly, our previous work elucidated that inhibiting class I HDACs with MS-275 is more beneficial in reducing injury in rat ischemia reperfusion models compared with using pan-HDAC inhibitors (24). Supplemental Figure 4 shows that MS-275 did promote significant increases of Wisp-1, specifically with LV myocardium at 7 days after MI; however, it did not promote significant increases of Vegf-a, as demonstrated with SAHA.

WISP-1 and its secretion are regulated through inhibition of HDACs. We wanted to evaluate the impact of HDAC inhibition-mediated WISP-1 expression in endothelial cells, since this cell type plays a major role in establishing vasculature. Human coronary artery endothelial cells (HCAECs) were treated with either PBS or TNF- $\alpha$ in the presence of $1-\mu \mathrm{M}$ and $5-\mu \mathrm{M}$ doses of SAHA. We used TNF- $\alpha$ since it is upregulated with the post-MI injury (42) and has been implicated to regulate Wisp-1 expression in murine isolated cardiomyocytes and fibroblast cells in vitro (12). We assessed the amount of WISP-1 found in both cell lysates, as well as the corresponding concentrated conditioned media (CM), for each experimental group, since 
A

Myocytes Fibroblasts Endothelial

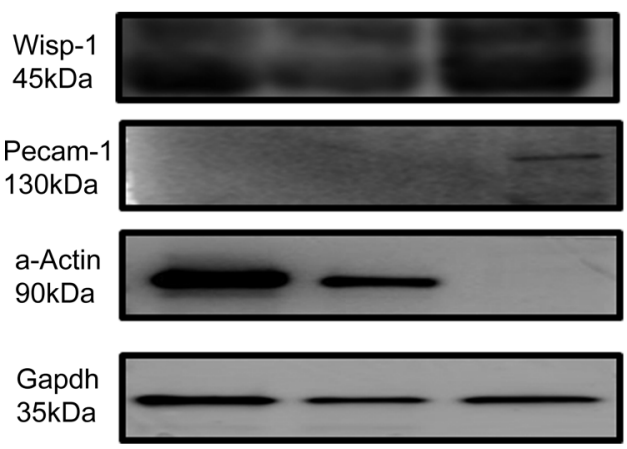

Rec. Wisp-1

B
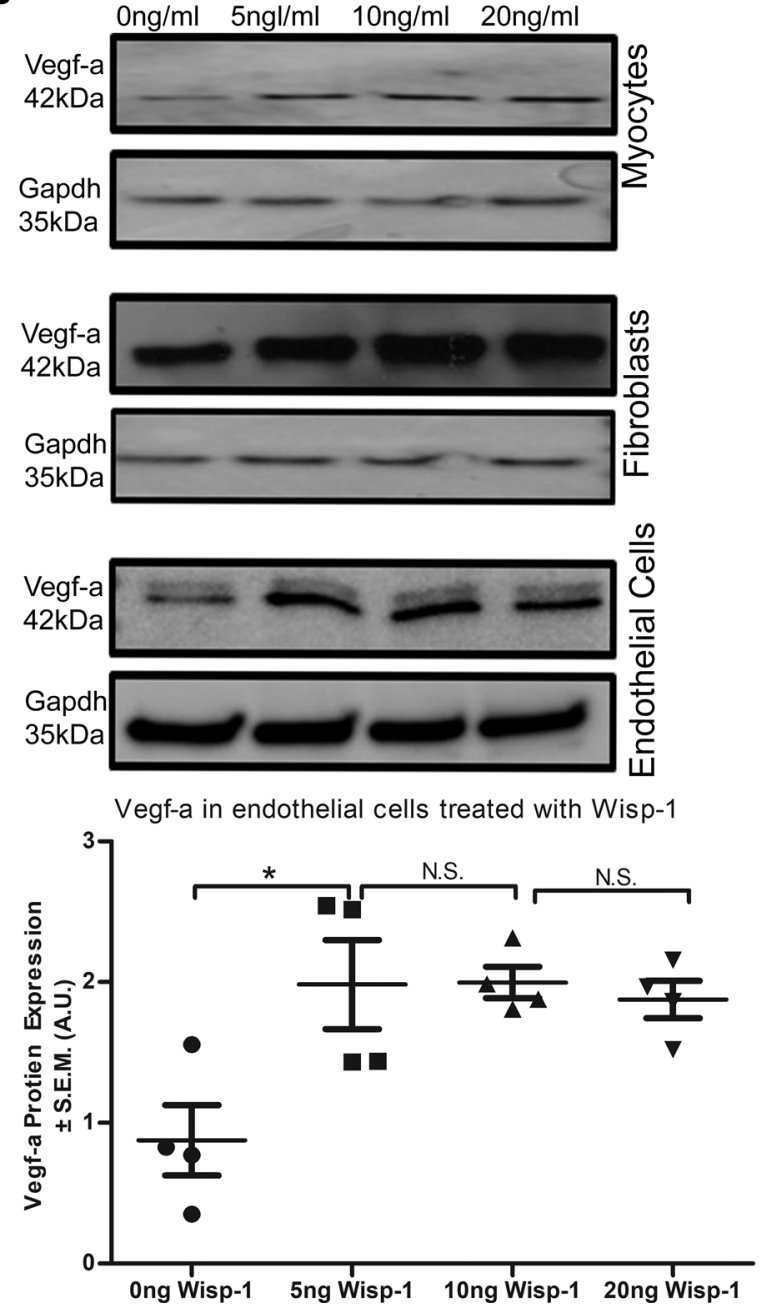

Wisp-1 protein

(Cells Isolated from whole LV)

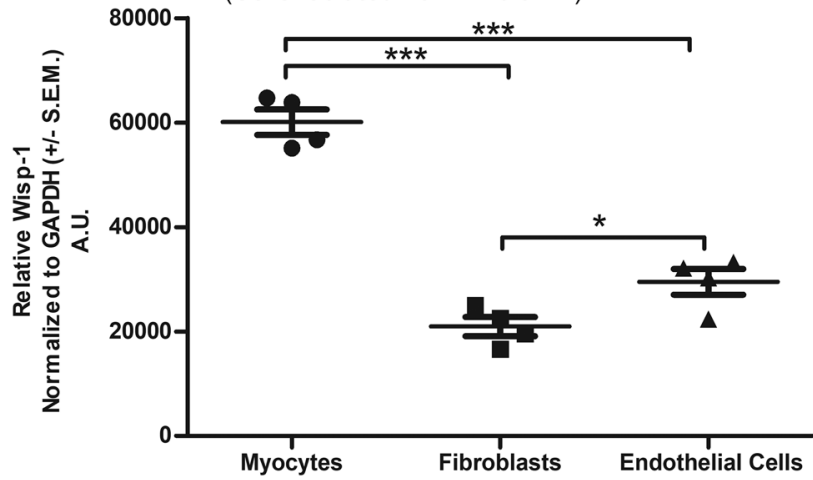

Vegf-a protein in myocytes treated with Wisp-1
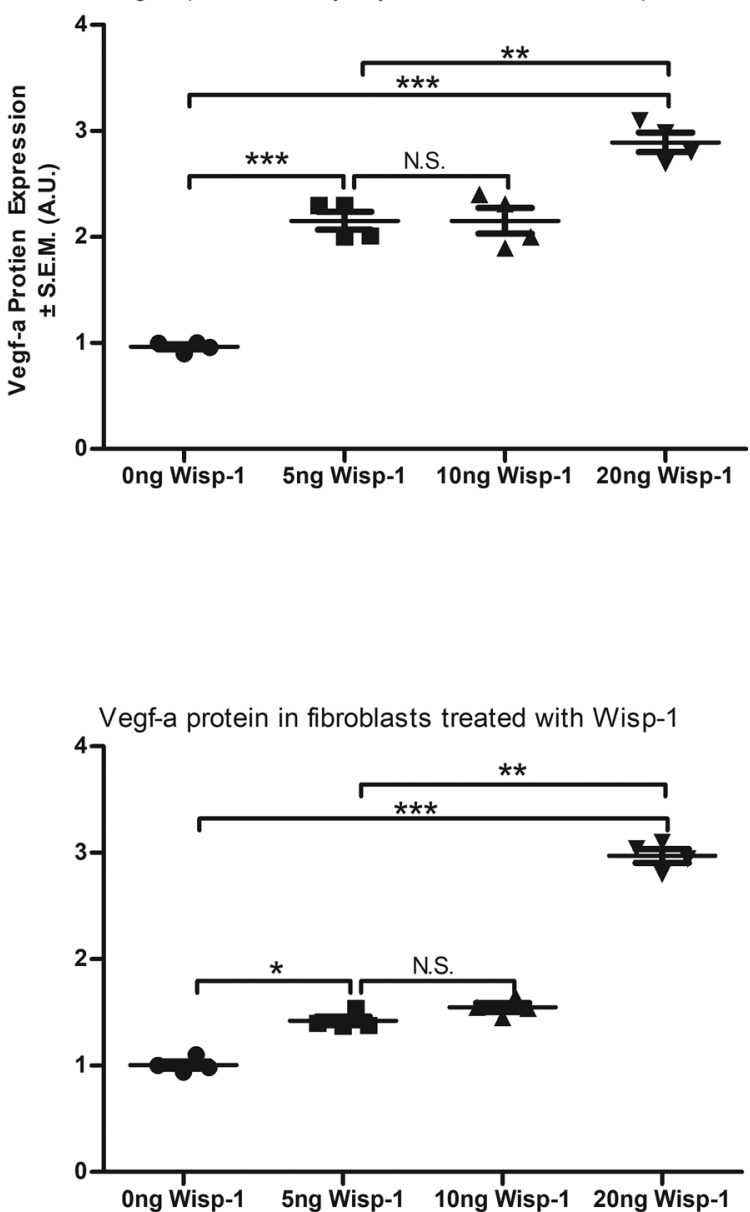

Figure 7. Isolated cardiac cells express Wisp-1 protein, and Wisp-1 itself directly enhances Vegf-a in these cells. (A) Western blot analysis of relative Wisp-1 detected in isolated murine cardiac cells ( $n=4$ independent cell isolations from left ventricle of normal male 10-week-old WT CD1 mice).

(B) Impact of recombinant Wisp-1 (rec.Wisp-1) treatment on Vegf-a production in isolated murine cells myocytes, fibroblasts, and endothelial cells. Equal numbers of cells were isolated and seeded immediately. Four to 6 hours after seeding, cells were gently washed 3 times with warm phosphate buffered solution and treated with $0 \mathrm{ng} / \mathrm{ml}, 5 \mathrm{ng} / \mathrm{ml}, 10 \mathrm{ng} / \mathrm{ml}$, or $20 \mathrm{ng} / \mathrm{ml}$ rec.Wisp-1 (murine) for 72 hours. Protein expression of Vegf-a (normalized to Gapdh protein) was done via Western blot. Graphs are representative of average fold change, relative to control $(0 \mathrm{ng} / \mathrm{ml})$ treatment. $\mathbf{A}$ and $\mathbf{B}$ are from 4 mice per group and are representative of 3 independent studies. Results depicted as mean $\pm \mathrm{SEM},{ }^{*} P \leq 0.05,{ }^{* *} P \leq 0.01,{ }^{* * *} P \leq 0.001$. $P$ values obtained by 1-way ANOVA with Tukey's post test. 

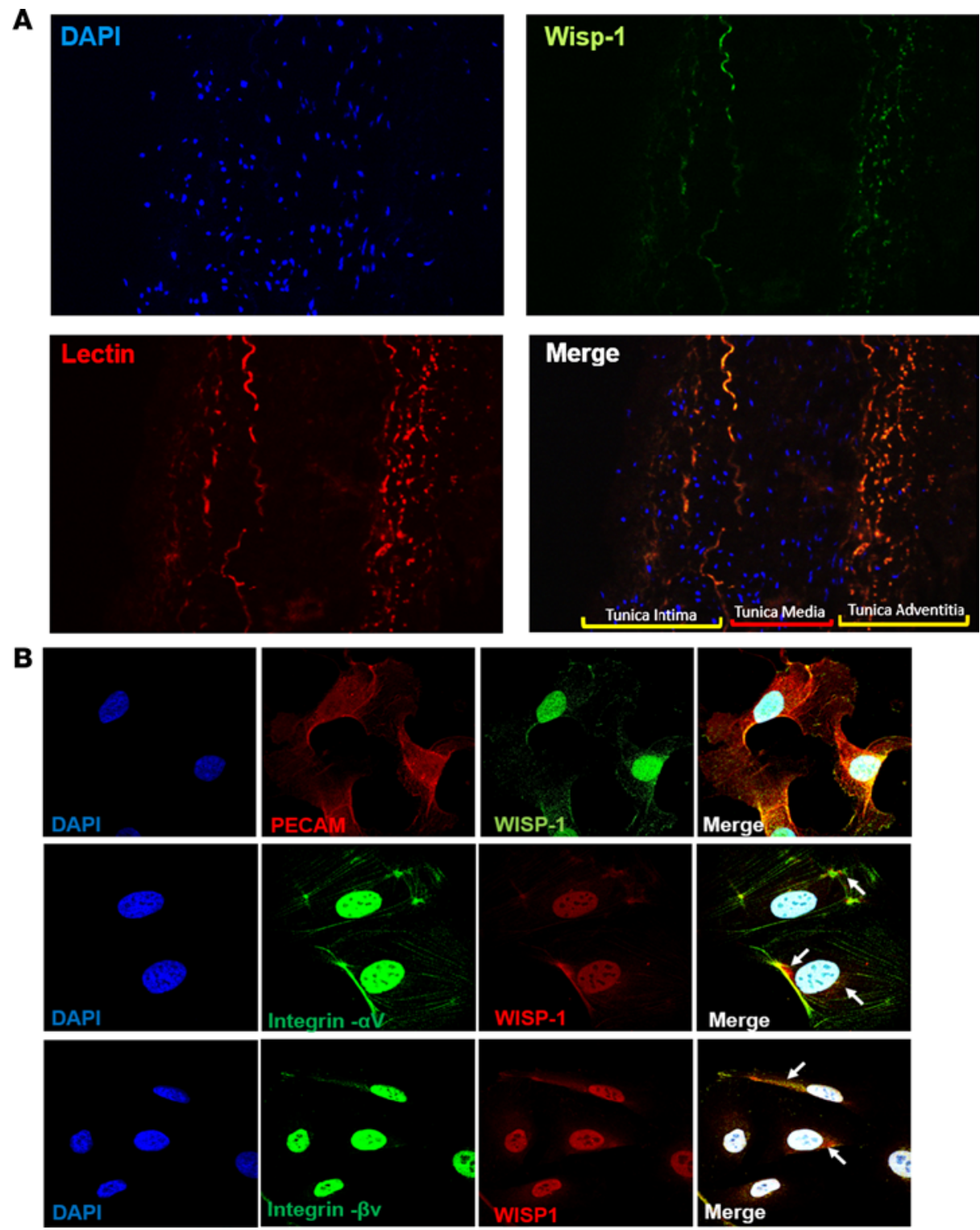

Figure 8. WISP-1 is detected within human coronary arteries and isolated human coronary artery endothelial cells. (A) Representative immunofluorescent images of WISP-1 (green) and lectin (red) in a normal/healthy intact human coronary artery, tunica intima, media, and adventitia are labeled in merged image. DAPI, 20x magnification. Image is representative of 3 repeated analyses. (B) WISP-1, PECAM-1, and integrins $\alpha \mathrm{V}$ and $\beta \mathrm{V}$ in isolated HCAECs, 60x, $n=$ 3 per experiment. White arrows highlight examples of colocalization of integrins and WISP-1.

WISP-1 is secreted. TNF- $\alpha$ alone significantly suppressed endogenous abundance of WISP-1 (Supplemental Figure 5) in HCAECs. However, treatment with $5 \mu \mathrm{M}$ SAHA in the presence of TNF- $\alpha$ resulted in an increase of WISP-1 relative to control, non-TNF- $\alpha$ stimulated levels when quantitating both lysate and CM (Supplemental Figure 5). These data are the first to our knowledge to report that SAHA directly promotes WISP-1 expression in TNF- $\alpha$-stimulated HCAECs.

HCAECs produce WISP-1 and integrins required for autocrine signaling. Although our data suggest that expression of WISP-1 can be regulated by HDAC inhibition, it is difficult to know whether WISP-1 itself contributes to a cardiac-specific angiogenic role as it does in cancer and neurological diseases. We examined lengthwise sections of human coronary arteries, which are composed of endothelial and smooth muscle cells, and found that WISP-1 is expressed and colocalizes with endothelial cell populations within the tunica intima and tunica adventitia (Figure $8 \mathrm{~A}$ ). We also assessed WISP-1 and the integrin receptors, $\alpha \mathrm{V}$ and $\beta \mathrm{V}$, since these integrins are known to illicit WISP-1-mediated downstream signaling $(37,43,44)$. HCAECs 
Human Coronary Artery Cells
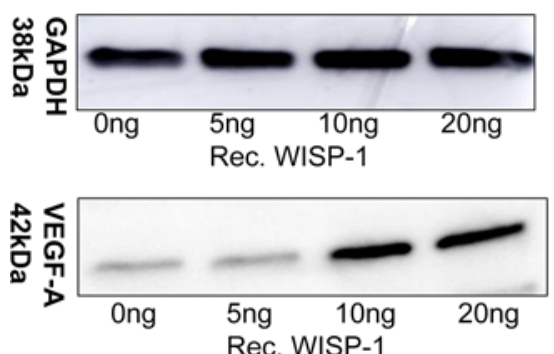

VEGF-A Protein Expression
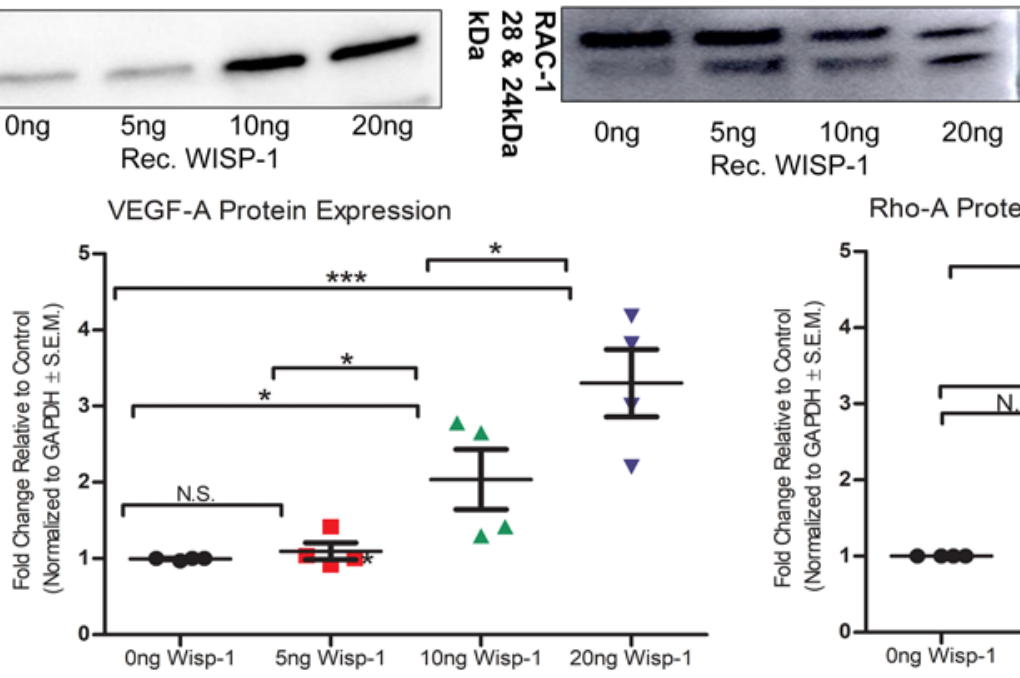
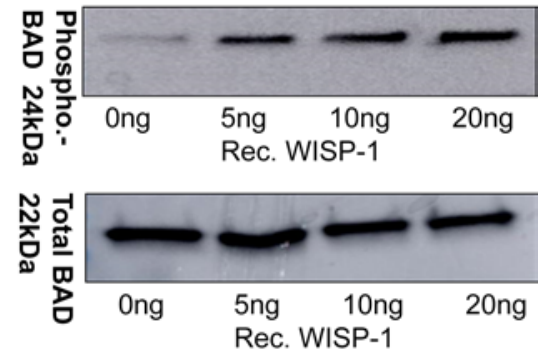

Rho-A Protein Expression
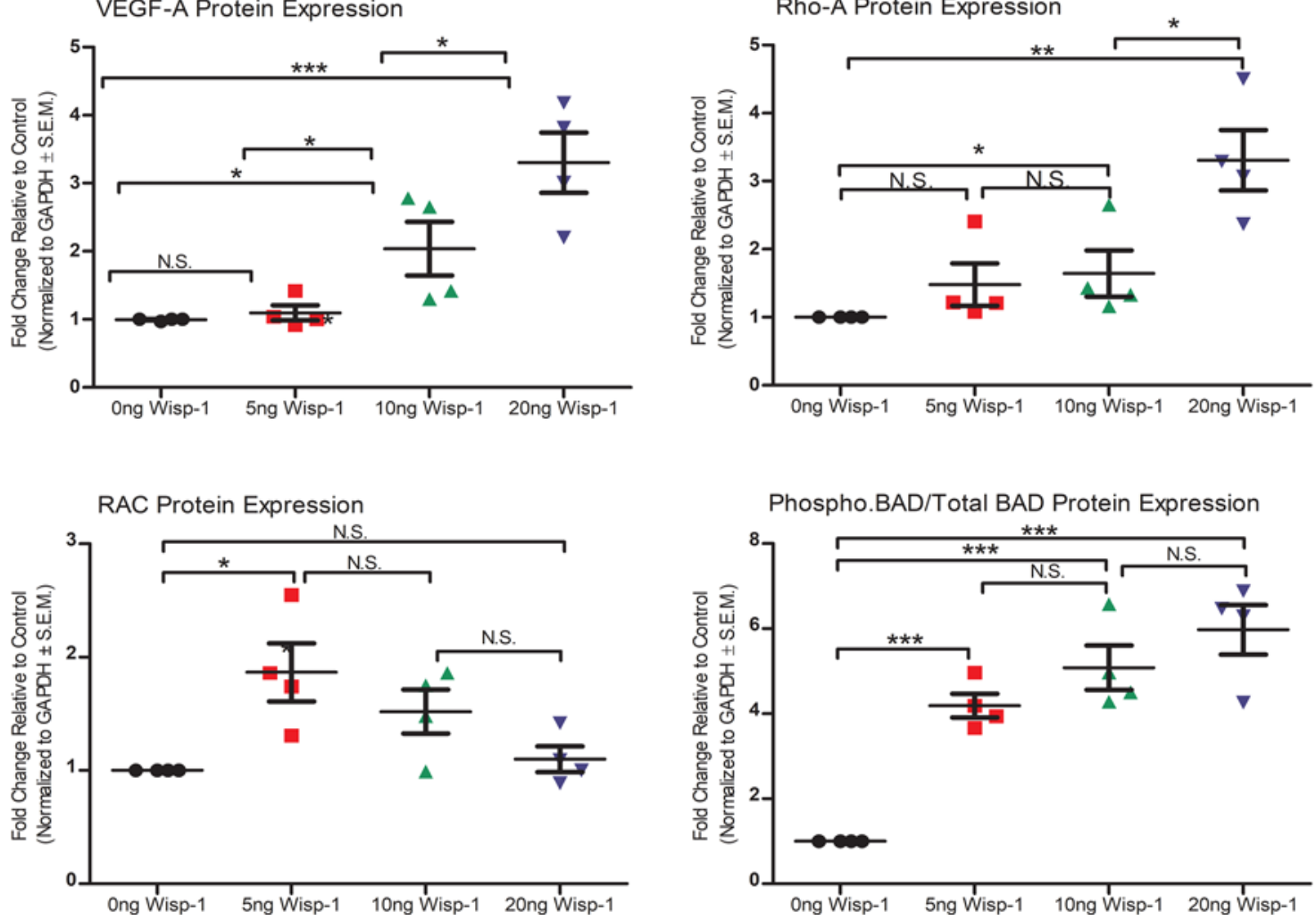

Figure 9. WISP-1 enhances proangiogenic and prosurvival genotypes in human coronary artery endothelial cells (HCAECs). Equal numbers of HCAECs were seeded overnight. The following day, cells were treated with $0 \mathrm{ng} / \mathrm{ml}, 5 \mathrm{ng} / \mathrm{ml}, 10 \mathrm{ng} / \mathrm{ml}$, or $20 \mathrm{ng} / \mathrm{ml}$ recombinant WISP-1 (rec.WISP-1) for 72 hours and then assessed for VEGF-A, Rho-A, RAC, the ratio of phospho-BAD/total BAD, and GAPDH (loading control) via Western blot. Graphs show quantitated densitometry differences normalized to loading control (GAPDH). Data is representative of 4 repeated analyses; results depicted as mean $\pm S E M$. ${ }^{*} P \leq 0.05$ relative to control, ${ }^{* *} P \leq 0.01,{ }^{* *} P \leq 0.001$. $P$ values obtained by 1-way ANOVA with Bonferroni's post test.

express Wisp-1 and the integrins required for autocrine signaling (Figure 8B). Importantly, immunofluorescent imaging show that WISP- 1 and $\alpha \mathrm{V}$ or $\beta \mathrm{V}$ are colocalized (Figure $8 \mathrm{~B} ; n=3$ magnification at $60 \times$ ). Interestingly, PECAM-1, which serves as an endothelial cell-specific marker, colocalized with WISP-1 throughout the cells (Figure 8B, top panel). Control cell staining with only fluorescent-labeled secondary antibodies (Alexa Fluor 488 and Alexa Fluor 568) and DAPI demonstrated a small degree of nuclear-specific autofluorescence; however, we did not observe nonspecific staining outside of nuclei in the cytoskeleton, which is where integrins would be located (Supplemental Figure 6).

WISP-1 promotes increased coronary artery endothelial cell proliferation, cell survival, and network density. Endogenous levels of WISP-1 within HCAECs were suppressed upon treatment with TNF- $\alpha$ but were significantly increased, even above baseline, with SAHA (Supplemental Figure 5). Therefore, we asked whether the presence of additional WISP-1, as seen in the LV-border zone, after MI through HDAC-mediated upregulation, impacts gene expression and the phenotypic characteristics of isolated HCAECs. To address this question, we evaluated the dose response of human rec.WISP-1 in HCAECs. Rec.WISP-1 significantly promoted the production of VEGF-A at higher concentrations (Figure 9). We then assessed the 

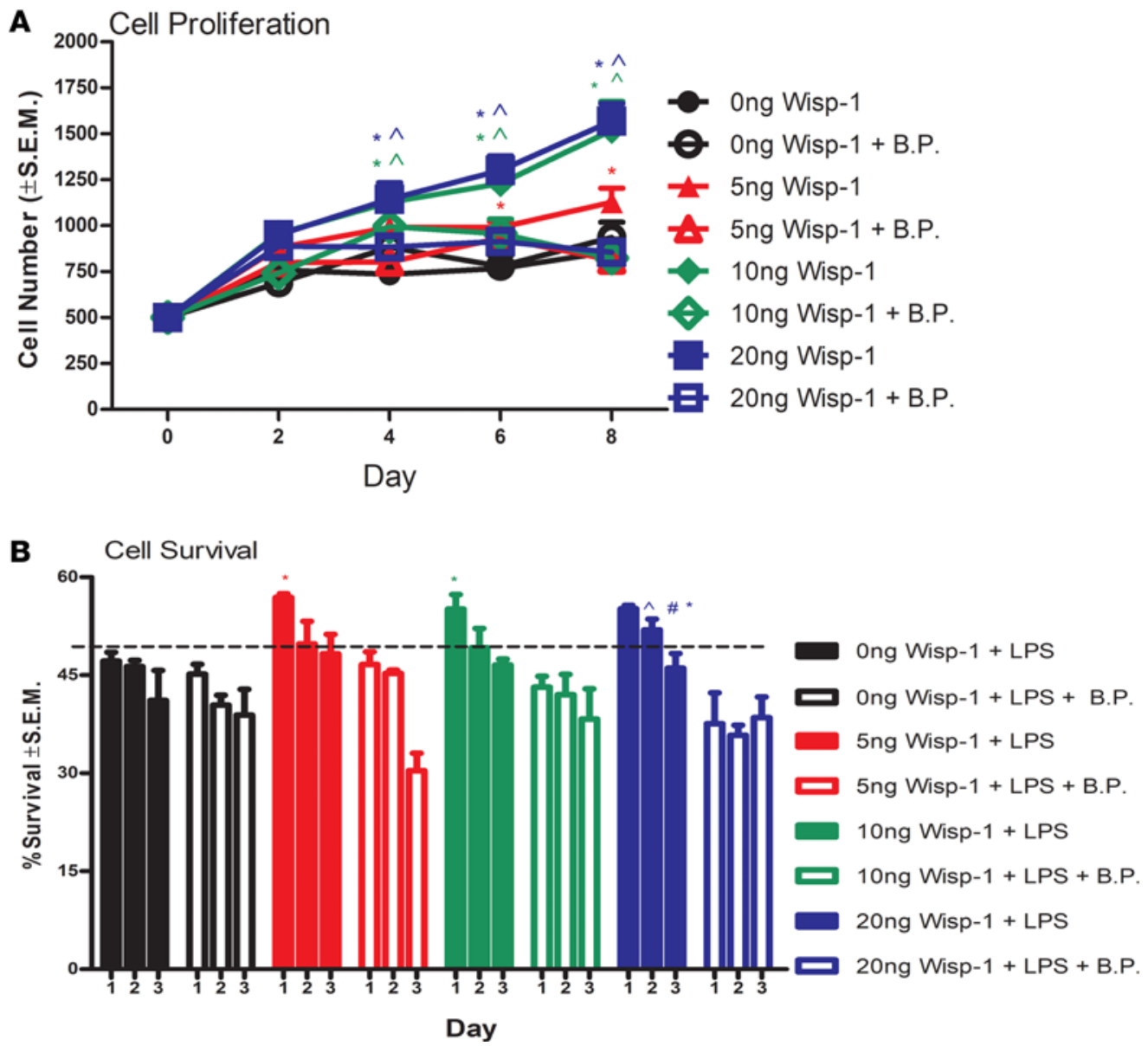

Figure 10. WISP-1 enhances proangiogenic behaviors in human coronary artery endothelial cells (HCAECs). (A) Two hundred fifty cells were seeded in a multiwell plate in triplicate in complete media. Cells were treated with either PBS $(0 \mathrm{ng} / \mathrm{ml}), 5 \mathrm{ng} / \mathrm{ml}$, and $10 \mathrm{ng} / \mathrm{ml}$, or $20 \mathrm{ng} / \mathrm{ml} \mathrm{rec}$. WISP-1 every 48 hours for 8 days. Cells were detached and counted on day 2, 4, 6, and 8 after seeding. (B)Five thousand cells were seeded in triplicate overnight in 24-well plates. The following day, cells were treated with either LPS $(10 \mu)$, rec.WISP-1, PBS ( $5 \mathrm{ng} / \mathrm{ml}, 10 \mathrm{ng} / \mathrm{ml}$, or $20 \mathrm{ng} / \mathrm{ml})$, and/or blocking peptide (BP, 1:1 of WISP-1). Cells were detached and counted manually every 24 hours for 3 days. A and B were performed in triplicate experimental groups and are representative of 3 independent experiments; results depicted as mean $\pm \mathrm{SEM},{ }^{*} P \leq 0.05$ relative to control, ${ }^{\wedge} P \leq 0.05$ relative to $5 \mathrm{ng} /$ $\mathrm{ml}, " \# \leq 0.05$ relative to $10 \mathrm{ng} / \mathrm{ml}$. A and $\mathbf{B} P$ values were obtained by 2 -way ANOVA.

expression of Rho-A, since Rho-A promotes endothelial cell aggregation required for proper vessel lumen formation (45). Similarly, WISP-1 only stimulated Rho-A expression (3-fold) at a $20 \mathrm{ng} / \mathrm{ml}$ dose, concentrations (Figure 9). Conversely, rec.WISP-1 suppressed the expression of RAC-1, a regulator of endothelial cell cytoskeletal arrangement and endogenous suppressor of angiogenesis (46), in a dose-dependent manner (Figure 9). Lastly, we assessed the impact of WISP-1 protein on the abundance and phosphorylation of the proapoptotic marker Bcl-2-associated death promoter (BAD) (47). Phosphorylation of BAD activates a prosurvival/antiapoptosis signaling mechanisms $(48,49)$. Importantly, WISP-1 has been shown to promote phosphorylation of BAD and subsequently help cells survive neurodegenerative conditions $(50,51)$. We did not see any significant changes in total BAD expression upon WISP-1 treatment; however, we did observe that phosphorylated-BAD increases up to 6-fold at 20ng/ml treatment of WISP-1 in HCAECs (Figure 9).

We next asked whether elevated expression of proangiogenic and prosurvival survival proteins by WISP-1 translates into phenotypic changes. All experimental doses of treatment with rec.WISP-1 promoted HCAEC proliferation, which were, in turn, functionally blunted by simultaneous treatment with a WISP-1 blocking peptide (BP) (Figure 10A). There were no significant differences in proliferation rates between 10 ng and 20 ng treatments, which suggests a threshold to WISP-1-mediated proliferative capacity (Figure 10B and Supplemental Figure 7). We then tested whether the increased phosphorylated-BAD due to WISP-1 translates into a prosurvival phenotype in HCAECs by simultaneously treating HCAECs with rec.WISP-1 
A $0 \mathrm{ng} / \mathrm{ml}$ WISP-1

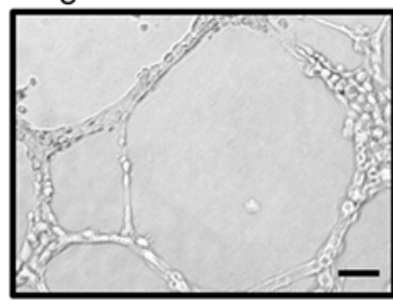

0ng/mI WISP-1 + B.P.

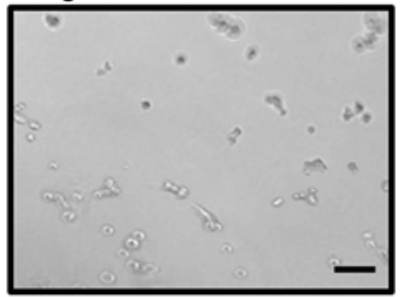

B

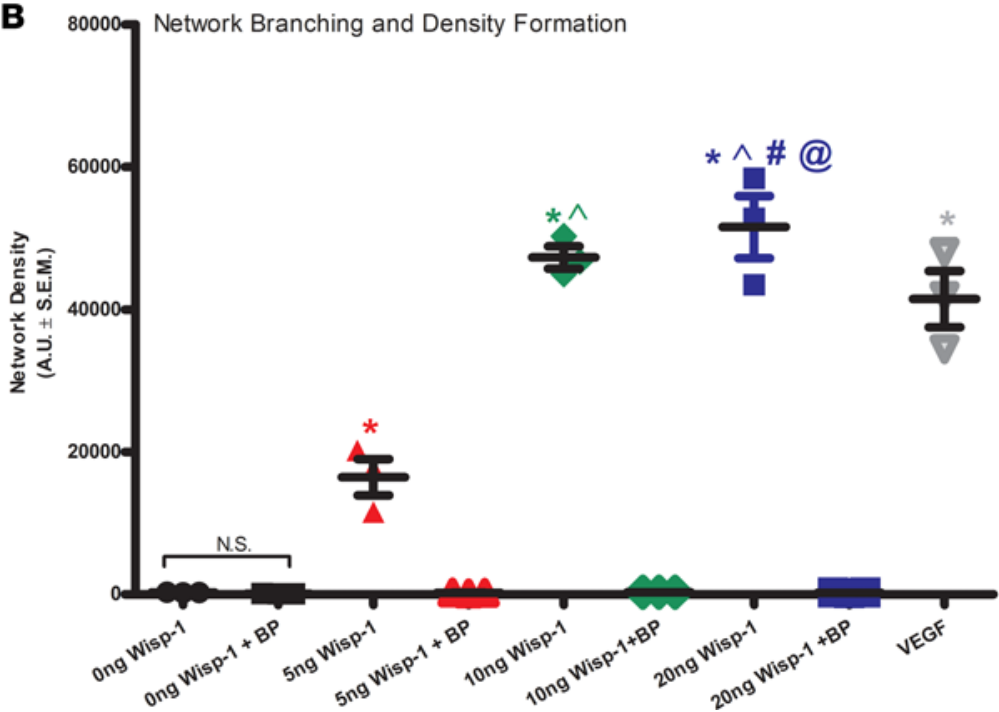

10ng/ml WISP-1

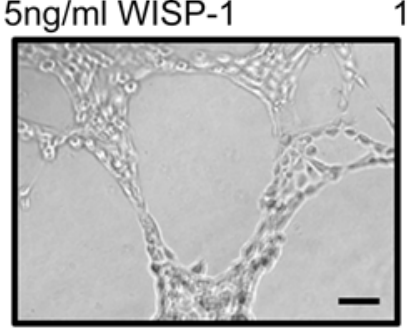

5ng/ml WISP-1 + B.P.

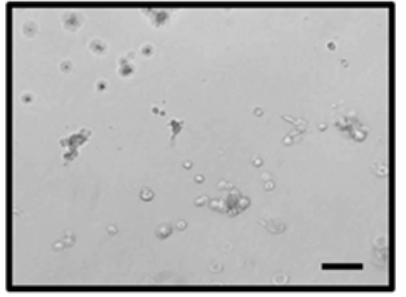

10ng/ml WISP-1 + B.P.
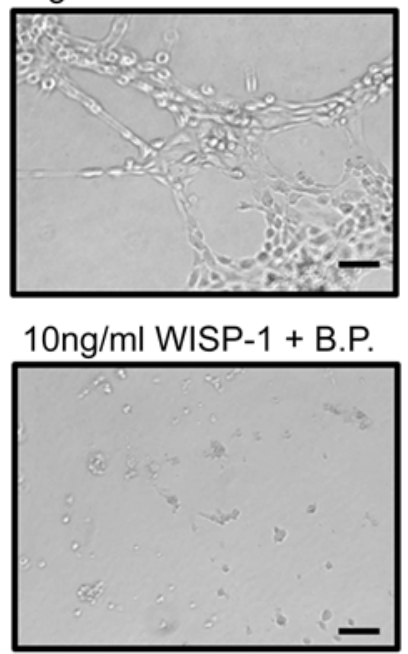

$20 \mathrm{ng} / \mathrm{ml}$ WISP-1



20ng/ml WISP-1 + B.P.

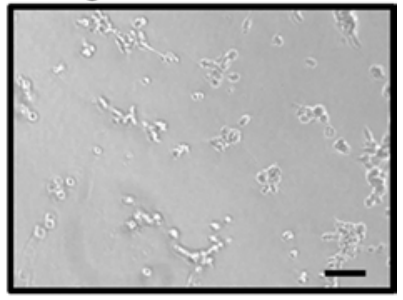

20ng/ml VEGF-A

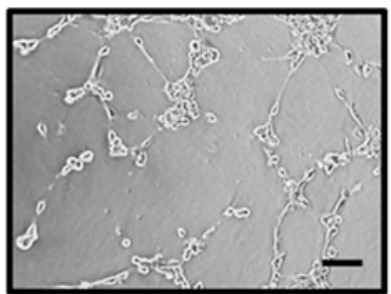

Figure 11. WISP-1 enhances endothelial cell branching and network densities in vitro. (A) Thirty thousand HCAECs were seeded in triplicate in 48 -well plates on growth factor-reduced Matrigel in the presence of $0 \mathrm{ng} / \mathrm{ml}$ (vehicle), $5 \mathrm{ng} / \mathrm{ml}, 10 \mathrm{ng} / \mathrm{ml}$, or $20 \mathrm{ng} / \mathrm{ml}$ recombinant WISP-1 and or BP, or $20 \mathrm{ng} / \mathrm{ml}$ VEGF-A. Scale bars: $100 \mu \mathrm{m}$. (B) Graph represents density of network branching determined by software analyses of images (5/well). A is representative of 4 repeated analyses. B was performed in triplicate of each experimental groups and are representative of 3 independent experiments. Results depicted as mean $\pm \mathrm{SEM},{ }^{*} P \leq 0.05$ relative to control, ${ }^{\wedge} P \leq 0.05$ relative to $5 \mathrm{ng} / \mathrm{ml}$, ${ }^{\#} P \leq 0.05$ relative to $10 \mathrm{ng} / \mathrm{ml}$, and ${ }^{\circledR} P \leq 0.05$ relative to VEGF-A (10ng/ml). $P$ values obtained by 1-way with Bonferroni's post test.

and the apoptosis inducing endotoxin LPS (52). We saw significant attenuation of LPS-induced cell death at day 1 after treatment in all groups that received WISP-1 (Figure 10B). However, 2 days after treatment, only cells given $20 \mathrm{ng} / \mathrm{ml}$ of rec.WISP-1 demonstrated a significant attenuation of death (Figure 10B). At day 3 , each experimental group showed a decreasing trend of cell survival regardless of WISP-1 treatment and or concentration (Figure 10B). Neutralizing WISP-1 BP prevented WISP-1-mediated cell survival at 24 and 48 hours (20 ng WISP-1 only) (Figure 10B). Data here demonstrate that WISP-1 can help to mediate cell survival, at least in HCAECs.

We next assessed whether WISP-1 impacts endothelial cell network formation and density in vitro. Rec.WISP-1 acted in a dose-dependent way to enhance HCAEC network formation and vessel density within 12 hours (Figure 11A). WISP-1 BP prevented network formation in every group, even in our controls that were incubated with $1 \mathrm{ng} / \mathrm{ml}$ of BP. In order to make sure that the network density we observed was relevant to known angiogenic, network formation inducers - and that the HCAECs were responsive to make networks in vitro - we also treated 1 experimental group with VEGF-A $(10 \mathrm{ng} / \mathrm{ml})$ as a positive 

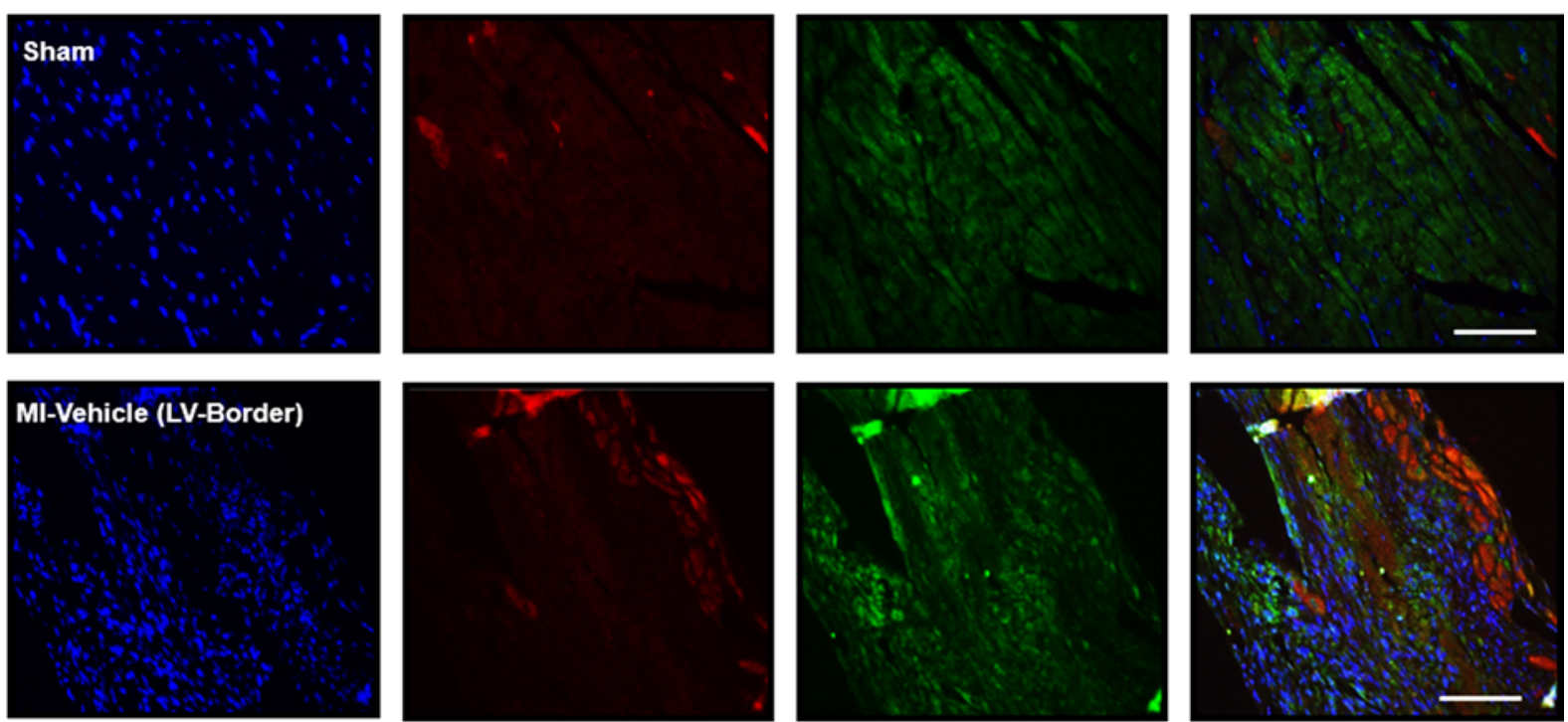

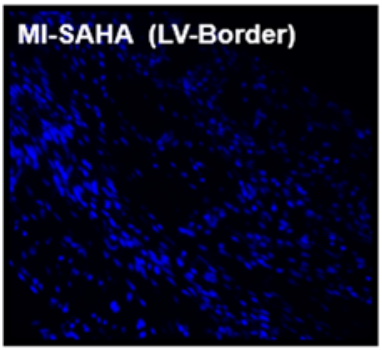

DAPI

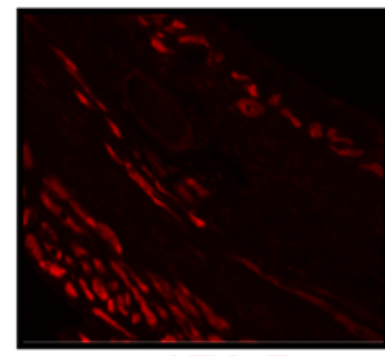

ATG-7

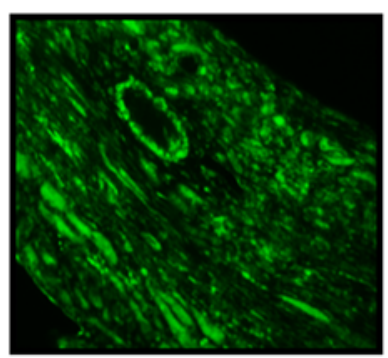

LC3

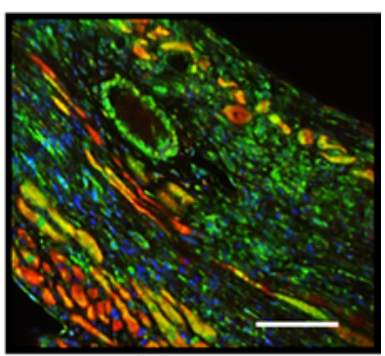

Merge

Figure 12. Inhibiting HDACs with SAHA enhances autophagy signaling in post-MI myocardium. Ten- to 12-week-old male CD1 mice were subjected to either sham (control) or ligation of the coronary artery surgery and received daily i.p. injections of DMSO (vehicle-control) or HDAC inhibition, SAHA (25 $\mathrm{mg} / \mathrm{kg}$ ). Seven days after MI, mice were euthanized. Localization and abundance of autophagy markers, ATG-7, and the LCZII complex in the border zone of infarction, $10 \times$ magnification, scale bars: $100 \mu \mathrm{m}$. Sham $n=6$ and MI $n=8$.

control. There were no statistical differences between endothelial cell network density with VEGF-A alone and or 10ng/ml WISP-1 treatment, $n=3, P \leq 0.05$ (Figure 11B). However, WISP-1 treatment of $20 \mathrm{ng} /$ $\mathrm{ml}$ showed branching densities that exceeded VEGF-A (Figure $11 \mathrm{~B}, P \leq 0.05$ relative to 10 ng VEGF). Together, these data suggest that WISP-1 promotes proangiogenic genotypic changes, which translate into angiogenic behaviors in vitro.

WISP-1 promotes autophagy signaling in HCAECs. HDAC inhibitors have been shown to play a role in regulating autophagy in heart disease $(53,54)$. Xie et al. have demonstrated that inhibiting HDACs and other acetylated proteins with inhibitors can attenuate adverse remodeling and infarct expansion associated with cardiac ischemia reperfusion injury models in rabbits by upregulating autophagy genes (32). Similarly to those findings, our data here demonstrate that broad HDAC inhibition with SAHA promoted increased abundance of autophagy markers ATG-7 and LC3 (Figure 12). We also assessed the direct impact of rec. WISP-1 on autophagy signaling in HCAECs, since SAHA indeed has many known and unknown targets. Interestingly, lower concentrations of rec.Wisp-1 significantly stimulated the increase of the ATG-5/ATG12 autophagy-promoting complex (Figure 13, $P<0.001, n=4$ /group). However, higher concentrations $(20 \mathrm{ng} / \mathrm{ml})$ of WISP-1 also stimulated approximately half-fold increase of this complex. We observed a similar trend with the ATG-7 marker in that concentrations up to $10 \mathrm{ng} / \mathrm{ml}$ of WISP-1 enhanced its abundance, while $20 \mathrm{ng} / \mathrm{ml}$ reduced its abundance. Rec.WISP-1 also promoted increased LC3B in both the 17 $\mathrm{kDa}$ and $14 \mathrm{kDa}$ fragments (Figure 13 and Supplemental Figure 8).

Constitutive regulation of endogenous WISP-1 expression alters network density behavior in HCAECs. Although we observed that treating HCAECs with human rec.WISP-1 protein enhances their angiogenic behaviors in vitro, we wanted to determine whether regulating endogenous WISP-1 in this cell population has any impact. Thus, we engineered HCAECs to constitutively express additional WISP-1 using human lentiviral 

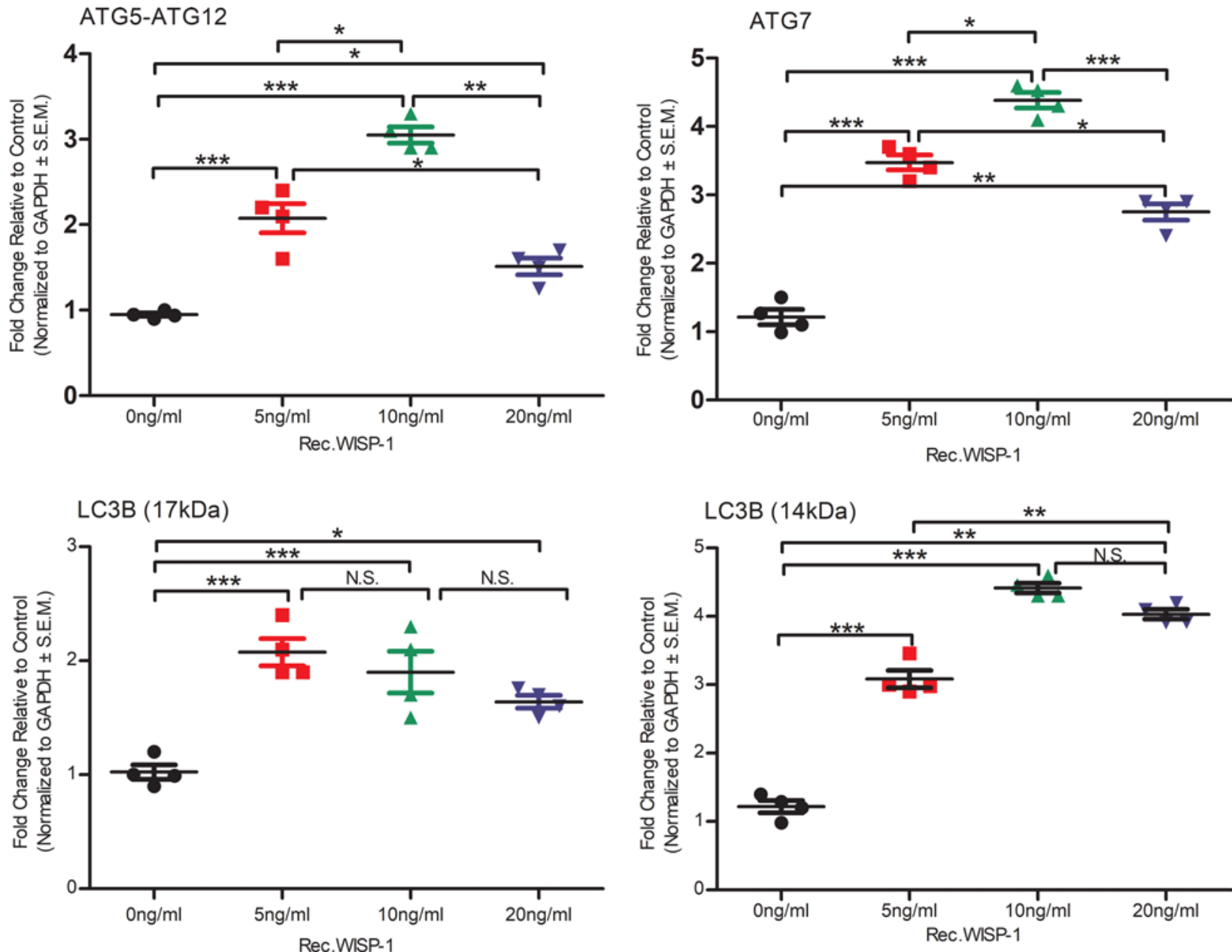

Figure 13. WISP-1 regulates autophagy signaling in human coronary artery endothelial cells (HCAECs). Equal numbers of HCAECs were seeded overnight. The following day, cells were treated with $0 \mathrm{ng} / \mathrm{ml}, 5 \mathrm{ng} / \mathrm{ml}, 10 \mathrm{ng} / \mathrm{ml}$, or $20 \mathrm{ng} / \mathrm{ml}$ recombinant WISP-1 (rec.WISP-1) for 72 hours and then assessed for autophagy markers ATC 5-ATC 12 complex, ATG-7, and LC3BII (17 and $14 \mathrm{kDa}$ ) and GAPDH (loading control). Graphs represent densitometry analyses of Western blots. $n=4$ per group. Results depicted as mean $\pm \mathrm{SEM},{ }^{*} P \leq 0.05,{ }^{* *} P \leq 0.01,{ }^{* *} P \leq 0.001$. $P$ values obtained by 1 -way ANOVA with Bonferroni's post test

activating particles (LVAP) (Figure 14A). We selected and expanded a clone (WISP-1-LVP-1) that had 4-fold constitutive induction of WISP-1 relative to cells expressing control/nontargeting lentiviral constructs, Ctrl-LVP-1 (Figure 14A, $n=3, P \leq 0.05$ relative to Ctrl-LVP-1). Cells engineered to constitutively overexpress WISP-1 significantly demonstrated enhanced cell branching network thickness relative to CtrlLVP-1 (Figure 14B). We evaluated whether TNF- $\alpha$, which we previously observed to suppress endogenous WISP-1 levels (Supplemental Figure 5), impacts network formation. We saw that TNF- $\alpha$ treatment significantly inhibited the WISP-1-mediated thickening of branches in both control and WISP-1-overexpressing cells (Figure 14B). However, the cells overexpressing WISP-1 demonstrated a significant enhancement of branching relative to control cells in the presence of TNF- $\alpha$ (Figure 14B).

In parallel, we also engineered HCAECs to constitutively suppress endogenous WISP-1 expression using shRNA technology (Figure 15A). Here, we selected and expanded clone sh-WISP-1-2, which showed an approximate 50\% knockdown of expression relative to nontargeting sh-RNA controls, termed, Ctrl-shRNA-1 (Figure 15A). Knocking down endogenous WISP-1 expression reduces branching thickness and density by almost one-third relative to Ctrl-shRNA-1 (Figure 15B). Again, we saw that treating the control group with TNF- $\alpha$ reduced network density abilities in vitro (Figure $15 \mathrm{~B}$ ). While there was a trend that constitutive WISP-1 knockdown had an even greater adverse impact on network density in the presence of TNF- $\alpha$, there was no significant difference between TNF- $\alpha$-treated Ctrl-shRNA-1 and sh-WISP-1-2 alone (Figure 15B). We attempted to isolate and amplify additional sh-Wisp-1 clones with constitutive knockdown of WISP-1 but failed to successfully propagate these 
A

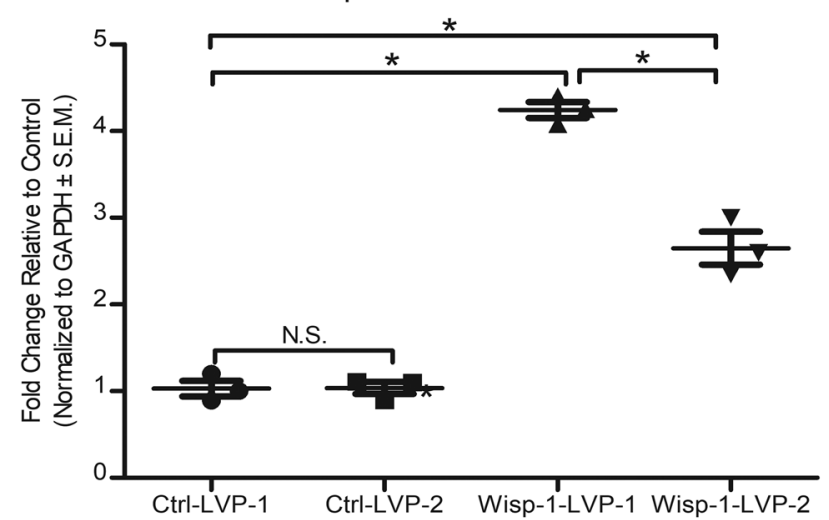

B

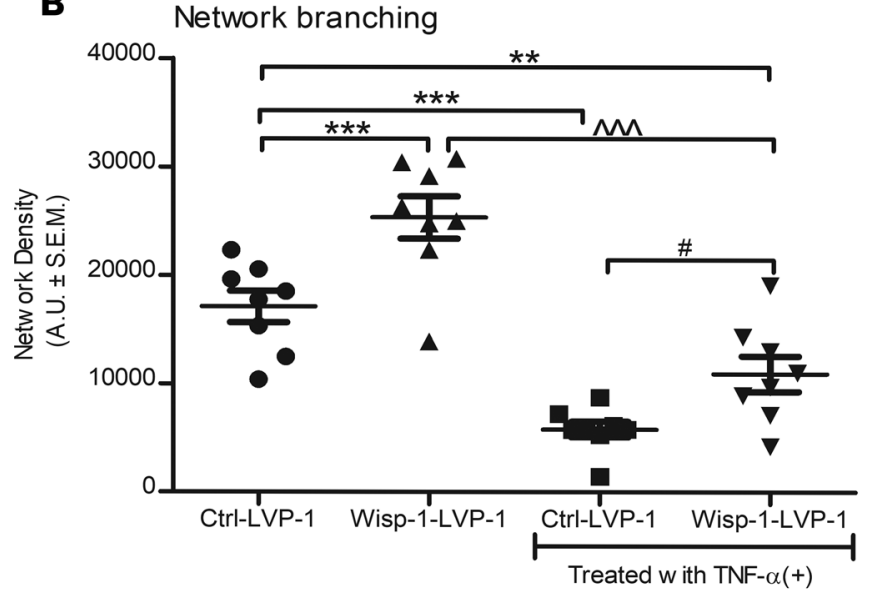

Lentivial Activating Particlemediated WISP-1
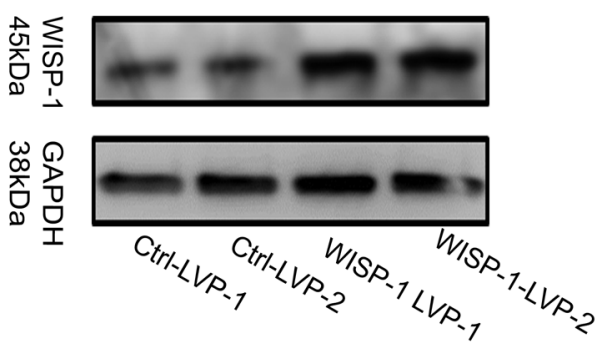

Ctrl LVP-1

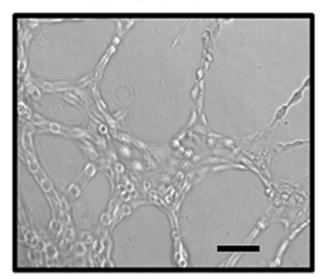

Ctrl LVP-1 +TNF- $\alpha$

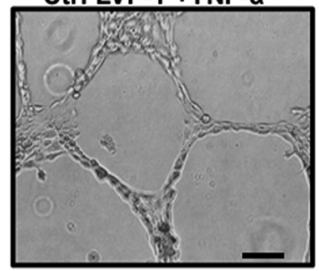

WISP-1-LVP-1

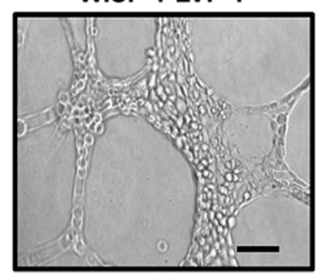

WISP-1-LVP-1 +TNF- $\alpha$

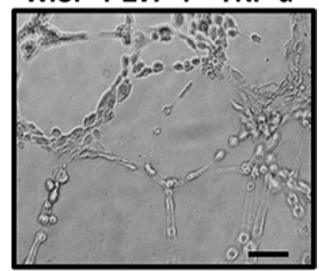

Figure 14. Constitutive overexpression of WISP-1 functionally enhances endothelial cell network density. (A) HCAECs were transduced with either control, nontargeting lentiviral activation particles (Ctrl-LVP), or WISP-1 lentiviral activation particles (WISP-1-LVP) for constitutive activation. (B) Clone, WISP-1-1 (most robust activation relative to control) was used to assess network density abilities. Thirty thousand cells from each experimental group were seeded in triplicate in 48-well plates on growth factor-reduced Matrigel. In parallel, cells were also treated with TNF- $\alpha$ (10 ng/ml). Density of network branching was determined by software analyses of images (5/well) after 8 hours. Graphs show branching density determined by software analyses. Analysis of Western blots are from the same experimental group, repeated 3 times. Data in $\mathbf{B}$ are from experiments performed in triplicate; $n=8$ per group (average density of 5 photographs per group). Results depicted as mean $\pm \mathrm{SEM}$ of AUs ${ }^{*} P \leq 0.05,{ }^{* *} P \leq 0.01,{ }^{* * *} P \leq 0.001$, relative to Ctrl-LVP-1; ${ }^{\wedge \wedge} P \leq$ 0.001 , relative to Wisp-1-LVP-1; ${ }^{*} P \leq 0.001$, relative to Ctrl-LVP-1 with TNF- $\alpha$. $P$ values obtained by 1-way ANOVA with Bonferroni's post test.

clones for further analysis. However, siRNA targets of WISP-1 demonstrated an approximate $50 \%$ knockdown of Wisp-1 in a mixed pool population of cells relative to both the control nontargeting siRNA and WT HCAECs (Supplemental Figure 9). As with shRNA-mediated knockdown seen in clone sh-Wisp-1-2 (Figure 15B), there was a significant reduction of network-forming capacity with loss of WISP-1/WISP-1 relative to nontargeting control $(P<0.001, n=15)$ and WT HCAECs $(P<0.001, n$ $=15)$ (Supplemental Figures 9 and 10). Furthermore, control cells treated with TNF- $\alpha$ demonstrated a significant reduction in network-formation capacity relative to untreated cells, while siRNA cells failed to show any networks in the presence of TNF- $\alpha$ (Supplemental Figures 9 and 10).

\section{Discussion}

This is the first report to our knowledge that demonstrates that the secreted molecule Wisp-1 is (i) upregulated in the border zone of infarction upon an acute MI ischemic injury, (ii) regulated by HDACs and can be upregulated through inhibition of HDACs via SAHA and MS-275, and (iii) a potent angiokine that regulates coronary artery-specific endothelial cells.

Our data demonstrate that Wisp-1/Wisp-1 is a transcriptional target of HDACs and is upregulated and increased, respectively, upon HDAC inhibition in vivo (Figure 3) and in isolated HCAECs (Supplemental Figure 5). In our in vitro studies, we used recombinant protein and RNAi to assess the impact of WISP-1 
A

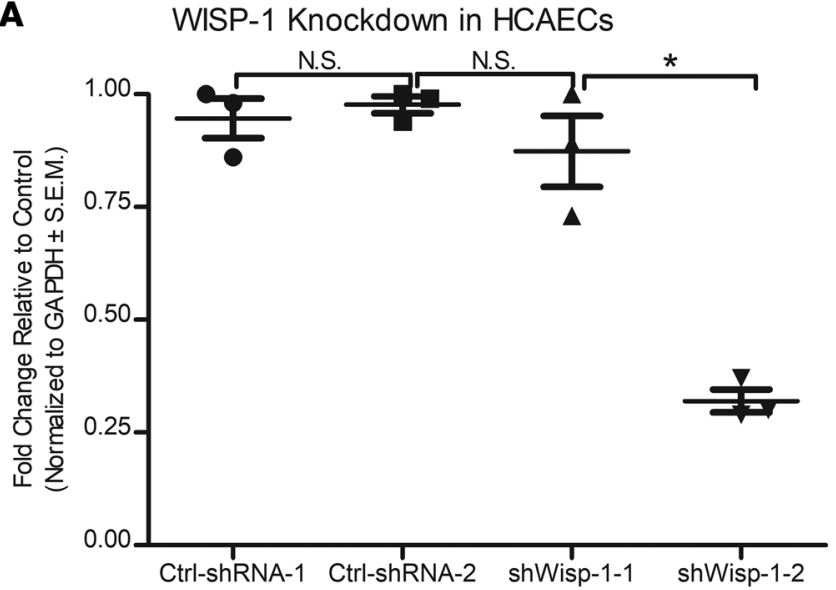

B

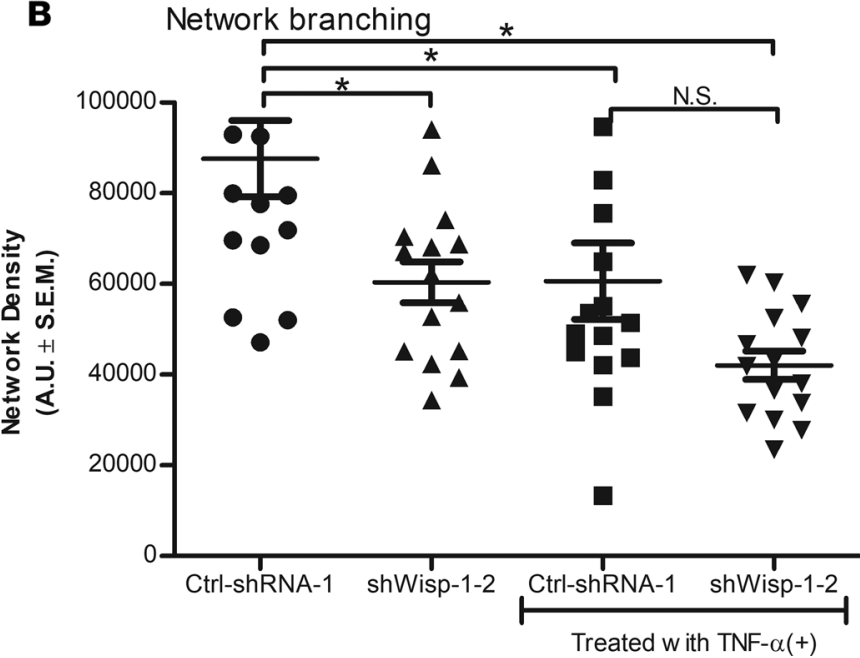

shRNA-mediated WISP-1

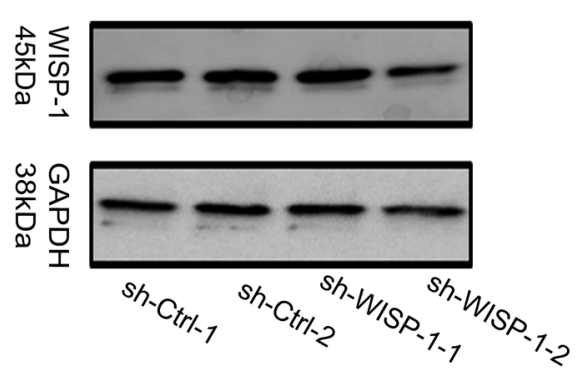

sh-Ctrl-1

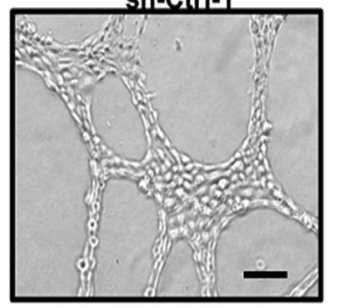

sh-Ctrl-1+TNF-a

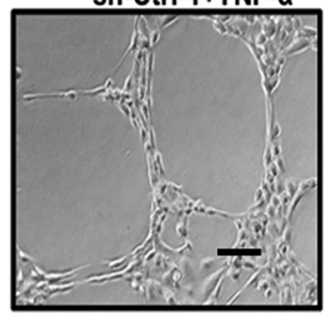

Sh-WISP-1-2

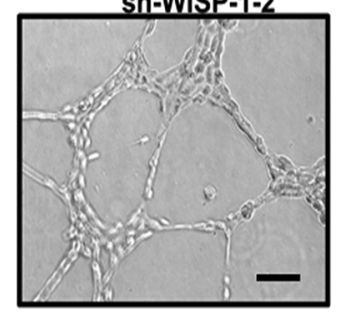

sh-WISP-1-2 +TNF-a

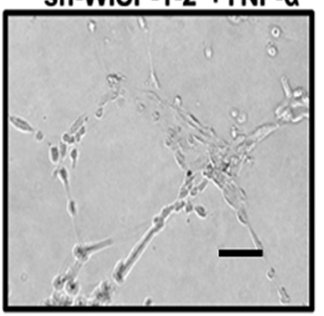

Figure 15. Constitutive downregulation of Wisp-1 functionally reduces endothelial cell network density. (A) HCAECs were transduced with lentiviral particles containing short hairpin-nontargeting, scramble control plasmids (sh-Ctrl), or Wisp-1 knockdown plasmids (sh-Wisp-1). (B) Clones with the most robust suppression of Wisp-1 were used to assess network density abilities. Thirty thousand cells from each experimental group were seeded in triplicate in 48-well plates on growth factor-reduced Matrigel. In parallel, cells were also treated with TNF- $\alpha$ (10 $\mathrm{ng} / \mathrm{ml})$. Density of network branching were determined by software analyses of images (5/well) after 8 hours. Graphs show branching density determined by software analyses. A is an analysis of Western blots from the same experimental group, repeated 3 times. B experiments were performed in triplicate with an $n=15$ per group (average density of 5 photographs per group). Results depicted as mean \pm SEM of AUs ${ }^{*} P \leq 0.05$. $P$ values obtained by 1-way ANOVA with Bonferroni's post test.

gain and loss of function on HCAECs. Our data show that WISP-1 can control network density and cell growth and survival. Cumulatively, we speculate that Wisp-1/Wisp-1 is upregulated by cardiomyocytes and other cell types within the post-MI myocardium in an attempt to create a proangiogenic microenvironment upon an ischemic injury. Furthermore, one of the reasons that treatment with SAHA demonstrates therapeutic benefits in small animal experimental models of $\mathrm{MI}$ is, in part, that it causes a significantly greater amount of Wisp-1, especially at the border zone of infarction. Furthermore, in vitro data assessment of human coronary artery cells demonstrate that adding rec.WISP-1 to their culture media can directly impact their survival, proliferation, expression of autophagy markers and vessel formation densities. Despite our potentially novel observations presented here, additional questions must be addressed for a more definitive understanding of what direct role WISP-1 might play in myocardium after an MI.

For example, we asked why an acute MI injury alone causes an upregulation of Wisp-1. One possible explanation is that Wisp-1 can be regulated by a variety of transcription factors, although activating complexes $\beta$-catenin/TCF/LEF are the most-well characterized and are therefore addressed in our research. Wang et al. have recently reported that Wisp-1 is upregulated by oxidative stresses such as ROS and prevents apoptosis through mitochondrial Bcl-2 signaling in neurodegenerative diseases (55). In these contexts, 
they found that Wisp-1 transcriptional expression is upregulated through the transcription factor FOXO3a, whose activity is sensitive to ROS (17). Considering that increased ROS are associated with acute MI injury (56), it is plausible that ROS within post-MI myocardium contribute to the initial upregulation of Wisp1 that we observed. Interestingly, our previous studies found that treatment with class I-specific HDAC inhibitor MS-275 causes an enrichment of nuclear FOX3a that correlated with reduced area of infarction in a rat model of cardiac ischemia reperfusion injury (24). However, in our studies here, MS-275-mediated increases of Wisp-1 suggest that MS-275 may be regulating Wisp-1 and other factors differently based on the injury model. However, it may be important to assess FOX3a and additional transcription factors that regulate Wisp-1 in an acute $\mathrm{MI}$ injury.

Another question that remains to be addressed is whether HDAC inhibitors are attenuating chromatin condensation around histones proximal to the promoter of Wisp-1, rendering a more transcriptionally available and active DNA region. Unfortunately, in the study presented here, we could not perform ChIP on more specific areas within post-MI myocardium; murine models yield very little tissue, and the ChIP technique requires $1-5 \mu \mathrm{g}$ of protein per antibody. It will be important to distinguish what role(s) Wisp-1 might play in remote noninfarct LV, infarcted, and border-zone regions; however, our in vitro data does suggest that perhaps it is regulating some facet of angiogenesis. That stated, more sensitive techniques, such as ChIP sequencing and proteomic assays that identify differentially acetylated targets, may help determine which factors are playing roles in regulating Wisp-1 expression after MI and, importantly, how HDAC inhibition might alter those factors, elucidating more therapeutic targets. It is plausible that multiple targets of WNT/Wisp-1 signaling are being regulated by HDAC inhibition, the impacts of which may have additive and or synergistic effects.

We detected Wisp-1 in the tunica intima and adventia, but not in the tunica media of undiseased human coronary arteries (Figure 8). Recent studies by Williams et al., however, show that Wisp-1 plays a role in the recruitment of vascular smooth muscle cells in atherosclerosis and, importantly, is expressed in the tunica media and intima only (57). Therefore, we are curious as to why we observed such differences in the subarterial localization of Wisp-1. We postulate that differences in reagents, specifically antibodies, and technique execution might partially explain the different localization of Wisp-1 expression. Another possible reason is that we assessed human coronary arteries, while Williams et al. (57) assessed human carotid arteries. Although there are similarities between both types of arteries, several studies have shown that each artery can respond to vascular-related pathologies differently based on temporal factors, age and sex, as reviewed in Jashari et al. (58). Perhaps the expression and subsequent function of Wisp-1 in the heart changes based on the discrete roles it plays within specific microenvironments. For example, earlier work from this same group of investigators show that restoring Wisp-1 in the carotid artery may prevent apoptosis-induced atherosclerotic rupture (59). Importantly, Williams et al. (57) and Mill et al. (59) have identified specific WNT activating ligands - Wnt2 and Wnt5a, respectively - that regulate expression of Wisp-1 in canonical and noncanonical WNT signaling. Further studies are needed to determine if there are specific WNT activating ligand(s) in the border zone of infarction that may regulate Wisp-1 expression and, importantly, whether they can be manipulated by HDAC inhibitors in regulating these ligands.

Another consideration for this work is the caveat that HDACs target many genes simultaneously, and subsequently, many genes may be impacted by HDAC inhibition. To delineate the role of Wisp-1 within post-MI myocardium, in vivo studies that use transgenic models to either conditionally overexpress or knock out Wisp-1 will be crucial in making relevant conclusions. Global KO of Wisp-1 in mice is nonlethal (60); however, we found that knockdown greater than $80 \%$ in adult HCAECs was lethal. We could not propagate a constitutive primary HCAEC cell line of total Wisp-1-KO; the cells failed to expand and did not survive subsequent cell culture passaging. We also saw, surprisingly, that neutralizing BPs functionally prevent successful network formation, even in control cells, by possibly blocking endogenous, autocrine Wisp-1 activity (Figure 11). These observations not only identify potential challenges with a KO murine model, but also underscore the seemingly crucial role that Wisp-1 plays in cardiac-specific blood vessel formation and stasis.

Another limitation within this study is that our in vivo data was performed using only male mice, and we assessed the myocardium 7 days after MI injury. Since estrogen is implicated to be cardioprotective in human and animal models of cardiovascular disease (61-63), we chose here to use male mice for initial investigations. However, Wisp-1 itself has been implicated in regulating several estrogen-driven mammary and gynecological cancers $(43,64,65)$. It will be essential and interesting, therefore, to 
determine whether Wisp-1 might play a different role in post-MI remodeling in female mice. In order to maintain continuity in sex considerations for the work done in this manuscript, we used HCAECs that were isolated from a male donor (see Methods). Again, it will be important to use both male and female isolated cells for more inclusive and comprehensive analyses of what role Wisp-1 plays in the post-MI myocardium and isolated cells.

It is also important to acknowledge that our studies evaluated Wisp-1 expression and abundance at 7 days after MI. It will be important to analyze Wisp-1 expression throughout the post-MI myocardium over both shorter and longer time periods. Temporal changes may play a significant role in determining when and how Wisp-1 is involved in post-MI remodeling. For example, Wisp-1 promotes increased collagen secretion in cardiac fibroblasts (12), pulmonary lung fibroblasts (66), and stromal cells within colon tumor lesions (15). However, it is unclear whether the collagen that is secreted specifically by stromal cells is in fact incorporated into the dynamic tissue-remodeling processes that occur several weeks after a MI. In consideration of our work presented here, it may be therapeutically helpful to quickly establish some level of fibrosis through elevated Wisp-1 expression in order to prevent rupture of a thinning ventricle while simultaneously promoting vessel formation to keep surrounding myocytes alive and abrogate infarct expansion. These considerations can only be assessed through studies that account for temporal monitoring throughout postMI events. Cumulatively, we suggest that Wisp-1 be considered for additional investigations directed at ameliorating acute-MI injury through promoting cell survival and enhancing microvasculature density in and around infarcted regions.

\section{Methods}

In vivo studies of ligation of the coronary artery. Male 10- to 12-week-old CD1 (Charles River Laboratories) mice were subjected to LAD coronary artery ligation to model an MI as previously described (67). After surgery, mice were injected (i.p.) daily (7 days) with either vehicle, dimethyl sulfoxide (DMSO), or the HDAC inhibitor SAHA (vorinostat; Selleckchem, catalog S1047) at $25 \mathrm{mg} / \mathrm{kg}$ in a total volume of 50 $\mu 1$ ( $n=12$ per group). Sham-operated mice served as surgical controls for our experiments $(n=8)$. Seven days after MI, cardiac physiology of end-diastolic volume and ejection fraction were evaluated (see Supplemental Methods for more details). An additional cohort of mice were injected with either PBS or class I HDAC inhibitor MS-275 (Entinostat; Selleckchem Houston, catalog S1053) at $5 \mathrm{mg} / \mathrm{kg}$ in a total volume of $50 \mu 1$ ( $n=4$ per group). LVs were excised and processed for further biochemical analyses. Surgical studies were repeated twice.

Isolation and treatment of cardiac-derived murine fibroblasts, myocytes, and endothelial cells. Male 10- to 15-week-old Sprague-Dawley rats (Charles River Laboratories) were injected i.p. with 1,000 U Heparin, which was allowed to circulate for 45 minutes. Rats were then anesthetized with $5 \%$ isoflurane prior to thoracotomy and heart excision. Excised hearts were subjected to aortic cannulation, followed by perfusion with $50 \mathrm{ml}$ of $37^{\circ} \mathrm{C}$ perfusion buffer (in mM: $113 \mathrm{NaCl}, 4.7 \mathrm{KCl}, 0.6 \mathrm{KH}_{2} \mathrm{PO}_{4}, 0.6 \mathrm{Na}_{2} \mathrm{HPO}_{4}, 1.2$ $\mathrm{MgSO}_{4}-7 \mathrm{H}_{2} \mathrm{O}, 12 \mathrm{NaHCO}_{3}, 10 \mathrm{KHCO}_{3}, 10 \mathrm{HEPES}, 6.3$ pyruvate, 30 taurine, 5.5 glucose, 10 2,3-butanedione monoxime, 0.9 adenosine, and 0.65 gadolinium chloride) to wash out residual blood. Hearts were then perfused with recirculating $37^{\circ} \mathrm{C}$ enzyme buffer (perfusion buffer with $0.1 \mathrm{mg} / \mathrm{ml}$ liberase blendzyme [Roche Diagnostics], $0.1 \mathrm{mg} / \mathrm{ml}$ trypsin [MilliporeSigma, and $12.5 \mathrm{nM} \mathrm{CaCl}_{2}$ ) for 45 minutes. Following digestion, ventricles were removed from the hearts and minced in perfusion buffer with $30 \%$ FBS to stop the digestion. Dispersion of cardiac cells was achieved by serial pipetting through serological pipettes, followed by sequential calcium reintroduction to a final concentration of $1.8 \mathrm{mM}$. Myocytes were separated from the other cell types by gravity sedimentation for 15 minutes. Endothelial cells and fibroblasts were further isolated via centrifugation and trypsin-digestion, as detailed in ref. 68. Chemicals were obtained from MilliporeSigma, unless otherwise indicated.

Cardiac-derived HCAECs. HCAECs were purchased from Cell Applications Inc. (catalog 300-5, lot 2989, normal human coronary arteries). Cells were expanded and frozen in liquid nitrogen until experimental use. Cells were cultured according to the manufacturer's recommendation at $37^{\circ} \mathrm{C}$ in humidified $\mathrm{CO}_{2}$ incubators Media was changed on cells every 3 days, and upon confluence, cells were detached with EDTA/trypsin and seeded into new Corning cell culture plates at ratios of 1:3 or 1:4. All experiments were performed within 5 subculture passages to maintain continuity of morphological and phenotypical characteristics.

Human blood vessel of the coronary artery. Premounted, 5- $\mu \mathrm{m}$ paraffin tissue sections of human coronary artery blood vessels were purchased from GeneTex Inc. (catalog GTX22224, lot 821603187). 
Cell proliferation assay. HCAECs (250 per experimental group) were seeded in triplicate overnight into a 96-well plate. The following morning, cells were treated with either 5, 10, or $20 \mathrm{ng} / \mathrm{ml}$ of human rec.WISP-1 (R\&D Systems, catalog 1627-WS-050) and or Wisp-1 BPs (Santa Cruz Biotechnology Inc., catalog sc-8866P) in a 1:1 ratio. Cells were detached and manually counted using a hemocytometer every 2 days for up to 8 days and supplemented with human rec.WISP-1 and or BPs on days 1, 3, 5, and 7 after seeding. Experiments were performed 3 times $(n=3)$.

Endothelial cell survival assay. HCAECs (5,000/well; 48 Multiwell Tissue Culture Plate (Corning Falcon, 353078) were seeded, in triplicate overnight. The next day, cells were treated with either $0 \mathrm{ng} / \mathrm{ml}$ (vehicle), $5 \mathrm{ng} / \mathrm{ml}, 10 \mathrm{ng} / \mathrm{ml}$, or $20 \mathrm{ng} / \mathrm{ml} \mathrm{rec}$.Wisp-1 and or Wisp-1 BP. Culture media was then supplemented with LPS (MilliporeSigma, $10 \mu \mathrm{g} / \mathrm{ml}$; ref. 69). Cells were detached and counted every 24 hours for 3 days. Experiments were performed in triplicate and repeated 3 times $(n=3)$.

Endothelial cell network/branching assay. CAECs (30,000/well; 48-well plate) were seeded in triplicate on growth factor reduced Matrigel, (Corning, catalog 356231). Cells were immediately treated with either $0 \mathrm{ng} / \mathrm{ml}$ (vehicle), $5 \mathrm{ng} / \mathrm{ml}, 10 \mathrm{ng} / \mathrm{ml}$, or $20 \mathrm{ng} / \mathrm{ml} \mathrm{rec.Wisp-1} \mathrm{and} \mathrm{or} \mathrm{Wisp-1} \mathrm{BP.} \mathrm{RNAi-based} \mathrm{network}$ branching studies differed in that cells from each experimental group were also treated with $10 \mathrm{ng} / \mathrm{ml}$ TNF- $\alpha$ (70) (R\&D Systems, catalog 210-TA-020/carrier free). After 8 hours, 5 images per well were taken of each experimental group using an inverted light Nikon TE microscope. Network density was quantified using ImageJ (Fiji), and data are represented as 3-D objects detected relative to background. Experiments were performed in triplicate and repeated 3 times $(n=3)$.

Quantitative PCR. Infarcted (MI) and corresponding healthy (sham) region of the mouse LV was removed, weighed, minced with scissors, and homogenized by polytron. RNA was isolated from these samples using an RNeasy Mini Fibrous Tissue Kit, (Qiagen, catalog 74704). A Bio-Rad iScript cDNA synthesis kit (Bio-Rad, catalog 1725038) was then used to convert the isolated RNA to cDNA. qPCR was performed using the Bio-Rad SsoAdvanced SYBR Green Supermix and primers for Wisp-1 and Gapdh (Bio-Rad, mouse Wisp-1, catalog 10025636, ID qMmuCID0018294; Gapdh, catalog 10025636, ID qMmuCED0027497). A Bio-Rad CFX96 Real Time Detection System was used to detect probe amplification, and the results were analyzed using the comparative Ct method. All data were normalized to the endorsed internal gene, Gapdh. Assays were performed in triplicate $(n=6)$.

Western blot analysis. Protein from cell lysates were quantified, and equal amounts of protein were resolved on SDS-PAGE by electrophoresis. Proteins were transferred onto a PVDF membrane and blocked with 5\% milk with TBST for 2 hours. Membranes were incubated with a primary antibody of interest; Wisp1, WISP-1 $\alpha$-actinin, PECAM-1, ATG 7, LC3B, BAD, Phospho-BAD, Histone H3, Rac-1, RhoA, VEGFA, $\alpha$-tubulin, Acetyl- $\alpha$-tubulin, and GAPDH (Supplemental Table 1)Membranes were washed and then incubated with an appropriate HRP-conjugated secondary antibody for 2 hours. Membranes were washed again and then incubated in diluted horseradish peroxidase substrate. Chromogenic changes were detected using AmerSham Imager 600 or ImageQuant LAS 4000 imagers (GE Healthcare) ( $n=3$ or 4).

Immunofluorescent chemical and immunocytochemical analyses. Tissue was fixed in paraformaldehyde and embedded in paraffin. Tissue was sectioned and mounted on glass microscope slides. Tissue was deparaffinized and rehydrated by sequentially increasing ratios of xylene, ethanol, and water. Antigen were exposed using sodium citrate buffer ( $\mathrm{pH}$ 6.0). Tissue was blocked in 10\% normal serum with 1\% BSA for 2 hours at room temperature. Slides were then incubated overnight with primary antibodies in a humidified $4^{\circ} \mathrm{C}$ chamber. Slides were washed and then incubated with fluorochrome-conjugated secondary antibodies and DAPI for 2 hours. Tissue was protected with coverslip and imaged using an Olympus 1X71 Slide Book-Inverted fluorescent microscope ( 5 images per heart/sample). The infarct scar was identified by fibrotic spaces that were void of nuclei. The border zones were identified by the tissue directly peripheral to the infarct areas. Remote zones were imaged from noninfarcted LV. Fluorescence intensity per field was determined using Image $\mathrm{J}$ (Fiji) and normalized to DAPI (nuclei). ( $n=6$, sham; $n=8, \mathrm{MI})$.

HCAECs were seeded on coverslips and permeabilized using triton X-100 (MilliporeSigma). Cells were incubated overnight with primary antibodies in a humidified $4^{\circ} \mathrm{C}$ chamber. Slides were washed and then incubated with fluorochrome-conjugated secondary antibodies and DAPI for 2 hours. Cells were covered with coverslips and imaged using an Olympus 1X81 FV10-ASW confocal microscope $(n=3)$.

Sirius red staining. In order to detect collagen, tissue was deparaffinized and rehydrated as described above. Slides were then stained in prefiltered Picrosirius red for 1 hour and washed twice in acidified water for 5 minutes. Slides were then dehydrated with 3 changes of ethanol and mounted with Richard-Allan 
Scientific Cytoseal (Thermo Fisher Scientific, catalog 8311-4). Five images (20× magnification) per heart were taken using an Olympus BX50WI Infinity Capture light microscope ( $n=6$, sham; $n=8, \mathrm{MI})$.

ChIP assay. ChIP analyses were performed as previously described (34). In brief, heart tissue was homogenized and protein-chromatin interactions were cross-linked, sonicated, and then lysed. Proteins of interested were immunoprecipitated using ChIP-grade antibodies (see Supplemental Methods); afterward, protein-chromatin complexes were reverse crosslinked, and chromatin was enriched using RNAase and Proteinase K. Chromatin associated with proteins on the proximal promoter of Wisp-1 was PCR amplified using primers: forward 5' - AATGCAGGGCTAGTCTGTTG - 3', reverse 5' - CTCCTCCCGCTGTTCATTATT - 3' (NCBI reference sequence NM_018865. GenBank Graphics >gi| 118130716:174-1277 Mus musculus WNT1 inducible signaling pathway protein 1 (Wisp1), mRNA).

Lentiviral particle transduction. Stable cell lines were generated using control lentiviral activation particles (Santa Cruz Biotechnology Inc., sc-437282) or Wisp-1 lentiviral activation particles (Santa Cruz Biotechnology Inc., sc-39335-V) (see Supplemental Table 2). Expression of Wisp-1 was validated using Western blot.

siRNA transfection. Human WISP-1 siRNA was purchased from Invitrogen (catalog AM16704, ID 137312, lot AS028HLJ). The scrambled siRNA was purchased as a control siRNA (catalog AM4611, lot ASO26WKF). HCAECs were cultured in 6-well plates and transfected with siRNAs (Wisp-1 siRNA and control siRNA; $20 \mathrm{nM}$ ) using Lipofectamine 2000 (Invitrogen, catalog 11668019) transfection reagent according to the manufacturer's instruction, followed by 48 hours of incubation in DMEM supplemented with $10 \% \mathrm{FBS}$ at $37^{\circ} \mathrm{C}$ in a $\mathrm{CO}_{2}$ incubator (see Supplemental Methods for sequences). Expression of Wisp1 was validated using Western blot.

Statistics. All studies were analyzed using either 1- or 2-way ANOVA. When appropriate, studies were further analyzed using either Tukey's or Bonferroni's multiple comparison post-test based on sample size per experimental group. In vitro assays were performed in either duplicate or triplicate and repeated at least 3 times. In vivo assays were performed 2 or 3 times with appropriate mice cohort numbers based on power analyses and previous studies. Data are represented as SEM. A value of $\mathrm{P}<0.05$ was considered statistically significant. Analyses were done using Graphpad Prism statistical software.

Study approval. Animal studies were approved by the Ralph H. Johnson-VA Affiliate Medical University of South Carolina IACUC. In vivo studies were granted approval from the VA-IACUC Animal Component of Research Protocol (ACORPS) committee (ACORPS approval 561).

\section{Author contributions}

LHW conceptualized the project. Methodology was designed by LHW. Experiments were performed by DJH (Figure 3A, Figure 7B, and Supplemental Figures 3 and 6), SSB (Figure 9), and HK (performed animal surgeries). LHW performed all other experiments. LHW and HK performed formal analyses. LHW wrote the original draft of manuscript. LHW, DJH, and DRM reviewed and edited the manuscript. Funding acquisition was by DRM. LHW and DRM provided resources.

\section{Acknowledgments}

We thank Denise Kimbrough and Jeff Jones who reviewed our manuscript and provided helpful critiques. Work was supported by the VA Merit BX002327 to DRM and the T32HL007260 to DRM. LHW was an NIH T32 fellow for the duration of this work. Additional support by F30HL129629 to DJH. We also thank those who allowed coauthors access to equipment.

Address correspondence to: Lillianne Harris Wright, Gazes Cardiac Research Institute, Medical University of South Carolina Room 331-STB; MSC 773, 114 Doughty Street, Charleston, South Carolina 294257730, USA. Phone: 843.696.3475; Email: harrislg@musc.edu or lilliwright100@gmail.com.

\footnotetext{
1. Mozaffarian D, et al. Heart disease and stroke statistics--2015 update: a report from the American Heart Association. Circulation. 2015;131(4):e29-322.

2. Cochain C, Channon KM, Silvestre JS. Angiogenesis in the infarcted myocardium. Antioxid Redox Signal. 2013;18(9):1100-1113.

3. Bialik S, et al. Myocyte apoptosis during acute myocardial infarction in the mouse localizes to hypoxic regions but occurs independently of p53. J Clin Invest. 1997;100(6):1363-1372.

4. Nadal-Ginard B, Kajstura J, Leri A, Anversa P. Myocyte death, growth, and regeneration in cardiac hypertrophy and failure. Circ Res. 2003;92(2):139-150.

5. Jugdutt BI. Ventricular remodeling after infarction and the extracellular collagen matrix: when is enough enough? Circulation.
} 
2003;108(11):1395-1403.

6. McKay RG, et al. Left ventricular remodeling after myocardial infarction: a corollary to infarct expansion. Circulation. 1986;74(4):693-702

7. Chu H, Wang Y. Therapeutic angiogenesis: controlled delivery of angiogenic factors. Ther Deliv. 2012;3(6):693-714.

8. Avolio E, et al. Combined intramyocardial delivery of human pericytes and cardiac stem cells additively improves the healing of mouse infarcted hearts through stimulation of vascular and muscular repair. Circ Res. 2015;116(10):e81-e94.

9. He W, et al. Exogenously administered secreted frizzled related protein 2 (Sfrp2) reduces fibrosis and improves cardiac function in a rat model of myocardial infarction. Proc Natl Acad Sci USA. 2010;107(49):21110-21115.

10. Frangogiannis NG. Matricellular proteins in cardiac adaptation and disease. Physiol Rev. 2012;92(2):635-688.

11. Wu RX, et al. Fibroblast migration after myocardial infarction is regulated by transient SPARC expression. $J$ Mol Med. 2006;84(3):241-252

12. Colston JT, et al. Wnt-induced secreted protein-1 is a prohypertrophic and profibrotic growth factor. Am J Physiol Heart Circ Physiol. 2007;293(3):H1839-H1846.

13. Chen CC, Lau LF. Functions and mechanisms of action of CCN matricellular proteins. Int J Biochem Cell Biol. 2009;41(4):771-783.

14. Chuang JY, et al. WISP-1 a novel angiogenic regulator of the CCN family promotes oral squamous cell carcinoma angiogenesis through VEGF-A expression. Oncotarget. 2015;6(6):4239-4252.

15. Pennica D, et al. WISP genes are members of the connective tissue growth factor family that are up-regulated in wnt-1-transformed cells and aberrantly expressed in human colon tumors. Proc Natl Acad Sci USA. 1998;95(25):14717-14722.

16. Alsarraf O, Fan J, Dahrouj M, Chou CJ, Menick DR, Crosson CE. Acetylation: a lysine modification with neuroprotective effects in ischemic retinal degeneration. Exp Eye Res. 2014;127:124-131.

17. Wang S, Chong ZZ, Shang YC, Maiese K. WISP1 neuroprotection requires FoxO3a post-translational modulation with autoregulatory control of SIRT1. Curr Neurovasc Res. 2013;10(1):54-69.

18. Wang J, Hu X, Jiang H. HDAC inhibition: A novel therapeutic target for attenuating myocardial ischemia and reperfusion injury by reversing cardiac remodeling. Int J Cardiol. 2015;190:126-127.

19. Cao DJ, et al. Histone deacetylase (HDAC) inhibitors attenuate cardiac hypertrophy by suppressing autophagy. Proc Natl Acad Sci USA. 2011;108(10):4123-4128.

20. McKinsey TA. The biology and therapeutic implications of HDACs in the heart. Handb Exp Pharmacol. 2011;206:57-78.

21. Davie JR. Histone modifications, chromatin structure, and the nuclear matrix. J Cell Biochem. 1996;62(2):149-157.

22. Bertos NR, Wang AH, Yang XJ. Class II histone deacetylases: structure, function, and regulation. Biochem Cell Biol. 2001;79(3):243-252.

23. Dai Y, Faller DV. Transcription Regulation by Class III Histone Deacetylases (HDACs)-Sirtuins. Transl Oncogenomics. 2008;3:53-65.

24. Aune SE, Herr DJ, Mani SK, Menick DR. Selective inhibition of class I but not class IIb histone deacetylases exerts cardiac protection from ischemia reperfusion. J Mol Cell Cardiol. 2014;72:138-145.

25. Granger A, et al. Histone deacetylase inhibition reduces myocardial ischemia-reperfusion injury in mice. FASEB J. 2008;22(10):3549-3560

26. Zhao TC, Cheng G, Zhang LX, Tseng YT, Padbury JF. Inhibition of histone deacetylases triggers pharmacologic preconditioning effects against myocardial ischemic injury. Cardiovasc Res. 2007;76(3):473-481.

27. Lee TM, Lin MS, Chang NC. Inhibition of histone deacetylase on ventricular remodeling in infarcted rats. Am J Physiol Heart Circ Physiol. 2007;293(2):H968-H977.

28. Xu L, Corcoran RB, Welsh JW, Pennica D, Levine AJ. WISP-1 is a Wnt-1- and beta-catenin-responsive oncogene. Genes Dev. 2000;14(5):585-595

29. Lévy L, et al. Acetylation of beta-catenin by p300 regulates beta-catenin-Tcf4 interaction. Mol Cell Biol. 2004;24(8):3404-3414

30. Virag JAI, Lust RM. Coronary artery ligation and intramyocardial injection in a murine model of infarction. $J$ Vis Exp. $2011 ;(52): 2581$

31. Kogan ME, Belov LN, Leont'eva TA, Zolotareva AG. [Modeling of myocardial pathology in mice with the surgical methods] Kardiologiia. 1977;17(6):125-128.

32. Xie M, et al. Histone deacetylase inhibition blunts ischemia/reperfusion injury by inducing cardiomyocyte autophagy. Circulation. 2014;129(10):1139-1151.

33. Chen Y, et al. Histone deacetylase (HDAC) inhibition improves myocardial function and prevents cardiac remodeling in diabetic mice. Cardiovasc Diabetol. 2015;14:99.

34. Harris LG, Wang SH, Mani SK, Kasiganesan H, Chou CJ, Menick DR. Evidence for a non-canonical role of HDAC5 in regulation of the cardiac Ncx1 and Bnp genes. Nucleic Acids Res. 2016;44(8):3610-3617.

35. Fischle W, et al. Enzymatic activity associated with class II HDACs is dependent on a multiprotein complex containing HDAC3 and SMRT/N-CoR. Mol Cell. 2002;9(1):45-57.

36. Wang Y, et al. Dysregulation of histone acetyltransferases and deacetylases in cardiovascular diseases. Oxid Med Cell Longev. 2014;2014:641979.

37. Wang S, Chong ZZ, Shang YC, Maiese K. WISP1 (CCN4) autoregulates its expression and nuclear trafficking of $\beta$-catenin during oxidant stress with limited effects upon neuronal autophagy. Curr Neurovasc Res. 2012;9(2):91-101.

38. Hecht A, Vleminckx K, Stemmler MP, van Roy F, Kemler R. The p300/CBP acetyltransferases function as transcriptional coactivators of beta-catenin in vertebrates. EMBO J. 2000;19(8):1839-1850.

39. Gao C, Xiao G, Hu J. Regulation of Wnt/ $\beta$-catenin signaling by posttranslational modifications. Cell Biosci. $2014 ; 4(1): 13$.

40. Hu E, et al. Identification of novel isoform-selective inhibitors within class I histone deacetylases. J Pharmacol Exp Ther. 2003;307(2):720-728.

41. Xu C, et al. Chemical probes identify a role for histone deacetylase 3 in Friedreich's ataxia gene silencing. Chem Biol. 2009;16(9):980-989.

42. Berthonneche $\mathrm{C}$, et al. New insights into the pathological role of TNF-alpha in early cardiac dysfunction and subsequent heart 
failure after infarction in rats. Am J Physiol Heart Circ Physiol. 2004;287(1):H340-H350.

43. Davies SR, Watkins G, Mansel RE, Jiang WG. Differential expression and prognostic implications of the CCN family members WISP-1, WISP-2, and WISP-3 in human breast cancer. Ann Surg Oncol. 2007;14(6):1909-1918.

44. Desnoyers L, Arnott D, Pennica D. WISP-1 binds to decorin and biglycan. J Biol Chem. 2001;276(50):47599-47607.

45. Bryan BA, et al. RhoA/ROCK signaling is essential for multiple aspects of VEGF-mediated angiogenesis. FASEB J. 2010;24(9):3186-3195.

46. Tan W, Palmby TR, Gavard J, Amornphimoltham P, Zheng Y, Gutkind JS. An essential role for Rac1 in endothelial cell function and vascular development. FASEB J. 2008;22(6):1829-1838.

47. Adams JM, Cory S. The Bcl-2 protein family: arbiters of cell survival. Science. 1998;281(5381):1322-1326

48. Zha J, Harada H, Yang E, Jockel J, Korsmeyer SJ. Serine phosphorylation of death agonist BAD in response to survival factor results in binding to 14-3-3 not BCL-X(L). Cell. 1996;87(4):619-628.

49. Fang X, et al. Regulation of BAD phosphorylation at serine 112 by the Ras-mitogen-activated protein kinase pathway. Oncogene. 1999;18(48):6635-6640.

50. Shang YC, Chong ZZ, Wang S, Maiese K. Wnt1 inducible signaling pathway protein 1 (WISP1) targets PRAS40 to govern ß-amyloid apoptotic injury of microglia. Curr Neurovasc Res. 2012;9(4):239-249.

51. Wang S, Chong ZZ, Shang YC, Maiese K. Wnt1 inducible signaling pathway protein 1 (WISP1) blocks neurodegeneration through phosphoinositide 3 kinase/Akt1 and apoptotic mitochondrial signaling involving Bad, Bax, Bim, and Bcl-xL. Curr Neurovasc Res. 2012;9(1):20-31.

52. Choi KB, Wong F, Harlan JM, Chaudhary PM, Hood L, Karsan A. Lipopolysaccharide mediates endothelial apoptosis by a FADD-dependent pathway. J Biol Chem. 1998;273(32):20185-20188.

53. Cao DJ, et al. Histone deacetylase (HDAC) inhibitors attenuate cardiac hypertrophy by suppressing autophagy. Proc Natl Acad Sci USA. 2011;108(10):4123-4128.

54. Zhang Y, Ren J. Targeting autophagy for the therapeutic application of histone deacetylase inhibitors in ischemia/reperfusion heart injury. Circulation. 2014;129(10):1088-1091.

55. Wang H, Zhang R, Wen S, McCafferty DM, Beck PL, MacNaughton WK. Nitric oxide increases Wnt-induced secreted protein-1 (WISP-1/CCN4) expression and function in colitis. J Mol Med. 2009;87(4):435-445.

56. Hildeman DA, Mitchell T, Aronow B, Wojciechowski S, Kappler J, Marrack P. Control of Bcl-2 expression by reactive oxygen species. Proc Natl Acad Sci USA. 2003;100(25):15035-15040.

57. Williams H, Mill CA, Monk BA, Hulin-Curtis S, Johnson JL, George SJ. Wnt2 and WISP-1/CCN4 Induce Intimal Thickening via Promotion of Smooth Muscle Cell Migration. Arterioscler Thromb Vasc Biol. 2016;36(7):1417-1424.

58. Jashari F, Ibrahimi P, Nicoll R, Bajraktari G, Wester P, Henein MY. Coronary and carotid atherosclerosis: similarities and differences. Atherosclerosis. 2013;227(2):193-200.

59. Mill C, et al. Wnt5a-induced Wnt1-inducible secreted protein-1 suppresses vascular smooth muscle cell apoptosis induced by oxidative stress. Arterioscler Thromb Vasc Biol. 2014;34(11):2449-2456.

60. Maeda A, et al. WNT1-induced Secreted Protein-1 (WISP1), a Novel Regulator of Bone Turnover and Wnt Signaling. J Biol Chem. 2015;290(22):14004-14018.

61. Booth EA, Obeid NR, Lucchesi BR. Activation of estrogen receptor-alpha protects the in vivo rabbit heart from ischemia-reperfusion injury. Am J Physiol Heart Circ Physiol. 2005;289(5):H2039-H2047.

62. Samsioe G. Cardioprotection by estrogens: implications of observational studies. Int J Fertil Menopausal Stud. 1994;39 Suppl $1: 20-27$.

63. Blenck CL, Harvey PA, Reckelhoff JF, Leinwand LA. The Importance of Biological Sex and Estrogen in Rodent Models of Cardiovascular Health and Disease. Circ Res. 2016;118(8):1294-1312.

64. Tang Q, et al. Expression and prognostic value of WISP-1 in patients with endometrial endometrioid adenocarcinoma. J Obstet Gynaecol Res. 2011;37(6):606-612.

65. Xie D, Nakachi K, Wang H, Elashoff R, Koeffler HP. Elevated levels of connective tissue growth factor, WISP-1, and CYR61 in primary breast cancers associated with more advanced features. Cancer Res. 2001;61(24):8917-8923.

66. Berschneider B, et al. miR-92a regulates TGF- $\beta 1$-induced WISP1 expression in pulmonary fibrosis. Int J Biochem Cell Biol. 2014;53:432-441.

67. Henry WL, et al. Report of the American Society of Echocardiography Committee on Nomenclature and Standards in Two-dimensional Echocardiography. Circulation. 1980;62(2):212-217.

68. Nistri S, Mazzetti L, Failli P, Bani D. High-Yield Method for Isolation and Culture of Endothelial Cells from Rat Coronary Blood Vessels Suitable for Analysis of Intracellular Calcium and Nitric Oxide Biosynthetic Pathways. Biol Proced Online. 2002;4:32-37.

69. Munshi N, Fernandis AZ, Cherla RP, Park IW, Ganju RK. Lipopolysaccharide-induced apoptosis of endothelial cells and its inhibition by vascular endothelial growth factor. J Immunol. 2002;168(11):5860-5866.

70. Venkatachalam K, et al. WISP1, a pro-mitogenic, pro-survival factor, mediates tumor necrosis factor-alpha (TNF-alpha)-stimulated cardiac fibroblast proliferation but inhibits TNF-alpha-induced cardiomyocyte death. J Biol Chem. 2009;284(21):14414-14427. 